\title{
Empirical validation of the Horowitz Multiple Systemic Infectious Disease Syndrome Questionnaire for suspected Lyme disease
}

This article was published in the following Dove Press journal:

International Journal of General Medicine

4 September 2017

Number of times this article has been viewed

\section{Maryalice Citera' \\ Phyllis R Freeman ${ }^{2}$ \\ Richard I Horowitz ${ }^{2}$ \\ 'Department of Psychology, State University of New York at New Paltz, New Paltz, NY, ${ }^{2}$ Hudson Valley Healing Arts Center, Hyde Park, NY, USA}

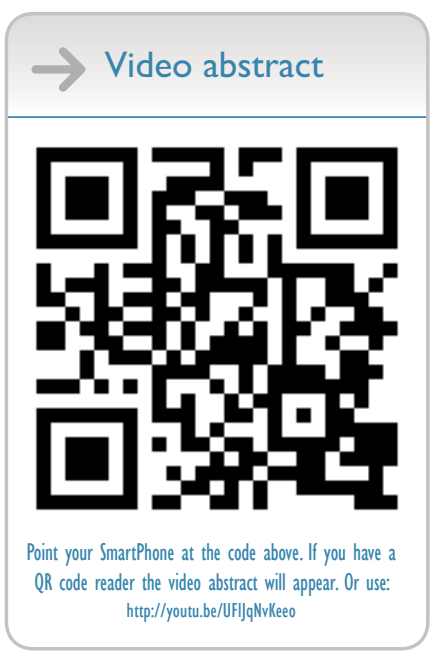

Correspondence: Maryalice Citera Department of Psychology, State University of New York New Paltz, I Hawk Drive, 353 Wooster Hall, New Paltz, NY 1256I, USA

Tel +l 8452573476

Fax +I 8452573470

Email citeram@newpaltz.edu
Purpose: Lyme disease is spreading worldwide, with multiple Borrelia species causing a broad range of clinical symptoms that mimic other illnesses. A validated Lyme disease screening questionnaire would be clinically useful for both providers and patients. Three studies evaluated such a screening tool, namely the Horowitz Multiple Systemic Infectious Disease Syndrome (MSIDS) Questionnaire. The purpose was to see if the questionnaire could accurately distinguish between Lyme patients and healthy individuals.

Methods: Study 1 examined the construct validity of the scale examining its factor structure and reliability of the questionnaire among 537 individuals being treated for Lyme disease. Study 2 involved an online sample of 999 participants, who self-identified as either healthy $(\mathrm{N}=217)$ or suffering from Lyme now ( $\mathrm{N}=782$ ) who completed the Horowitz MSIDS Questionnaire (HMQ) along with an outdoor activity survey. We examined convergent validity among components of the scale and evaluated discriminant validity with the Big Five personality characteristics. The third study compared a sample of 236 patients with confirmed Lyme disease with an online sample of 568 healthy individuals.

Results: Factor analysis results identified six underlying latent dimensions; four of these overlapped with critical symptoms identified by Horowitz - neuropathy, cognitive dysfunction, musculoskeletal pain, and fatigue. The HMQ showed acceptable levels of internal reliability using Cronbach's coefficient alpha and exhibited evidence of convergent and divergent validity. Components of the HMQ correlated more highly with each other than with unrelated traits.

Discussion: The results consistently demonstrated that the HMQ accurately differentiated those with Lyme disease from healthy individuals. Three migratory pain survey items (persistent muscular pain, arthritic pain, and nerve pain/paresthesias) robustly identified individuals with verified Lyme disease. The results support the use of the HMQ as a valid, efficient, and low-cost screening tool for medical practitioners to decide if additional testing is warranted to distinguish between Lyme disease and other illnesses.

Keywords: Lyme disease, Borrelia burgdorferi, Babesia, MSIDS, Multiple Systemic Infectious Disease Syndrome, factor analysis, PTLDS, Post-Treatment Lyme Disease Syndrome

\section{Background and overview}

Identification of individuals with Lyme disease is a major health concern. Lyme disease is caused by the bacteria, Borrelia burgdorferi $(\mathrm{Bb})$, which has one of the most complex genomes of any bacteria. ${ }^{1}$ Identifying Borrelia has proven challenging because it has the ability to evade the immune system ${ }^{2}$ and "the bacteria is able to traverse the blood brain barrier, endothelial tissue, and imbed itself in joints, entering certain cells intercellularly and invaginating itself in a manner that reduces the potential exposure of antigens, enabling it to avoid immune recognition". ${ }^{1}$ 
Lyme disease, already a significant public health threat in the United States and Europe, ${ }^{3,4}$ is poised to be the number one spreading vector-borne epidemic worldwide. Pathogencarrying ticks ride migratory birds across wide geographic areas spreading the infection. ${ }^{5}$

A recent study released by the Centers for Disease Control and Prevention (CDC $)^{6}$ identified a $320 \%$ increase in reported counties with Lyme disease in the northeastern United States. In the north-central states for the same periods, the number of counties having high incidence increased by $>250 \%$ with Lyme moving "northward and southward." Their conclusion was that "relatively constant rates of geographic expansion [were happening] in all accessible directions".

WHO European data report a steady increase in Lyme disease cases over the past 2 decades, with $>360,000$ cases having been reported. ${ }^{7}$ This may represent an underreporting of true incidence, as suggested by Sykes, who reported 232,125 cases in 1 year in Western Europe. ${ }^{8}$ The CDC estimated $>300,000$ new infections per year in the United States - just for Lyme - one of the several tick-borne diseases. ${ }^{9}$ The CDC estimates do not take into account those cases that are not reported or are misdiagnosed as other medical conditions. In 2012, ${ }^{10}$ an estimated $0.3 \%$ of the United States population were diagnosed, in that year alone, with Lyme disease - well over 900,000 people.

A 2015 study highlighted how Lyme disease patients with long-term complaints increased the burden of costs. Study authors ${ }^{11}$ looked at the actual costs of treating US patients in the year following their Lyme diagnosis and reported that "regardless of what you call it, our data show that many people who have been diagnosed with Lyme disease are in fact going back to the doctor complaining of persistent symptoms, getting multiple tests and being retreated." On average, "people with Lyme disease cost the system \$2,968 more than matched controls, and they cost the healthcare system about $\$ 1$ billion a year." 11

Previous published scientific studies showed even higher health care costs for treating Lyme disease. In one study, conducted in $2006^{12}$ and adjusted in 2013 figures for inflation, ${ }^{13}$ researchers found that the mean annual cost of illness was $\$ 20,502$ per year per patient for late Lyme disease, year after year. These costs might be much higher as suggested by a population-based cross-sectional study. ${ }^{14}$ In this study, Ahern concluded that the $\mathrm{CDC}$ was underestimating the risk of Lyme disease and the spread of borreliosis. European data also demonstrate that the cost-of-illness of Lyme disease is substantial compared to other infectious diseases ${ }^{15}$ and can cause significant disability. ${ }^{16}$
Diagnosing Lyme is an important worldwide problem and challenge for the medical community since the symptoms of tick-borne illness often mimic those of other commonly reported diseases that are diagnosed based on clinical criteria and not definitive laboratory testing including fibromyalgia, chronic fatigue syndrome (systemic exertional intolerance disease), multiple sclerosis, mental illness, and many others. ${ }^{17}$ Ahern's figures imply that millions of people per year are being infected with Lyme disease, and by the CDC's calculations, the true incidence is likely underestimated. ${ }^{18}$

Early diagnosis and treatment lead to better health outcomes and, thus, can be more cost effective. Unfortunately, current screening techniques are ineffectual, particularly for early detection.

\section{Testing for Lyme disease}

Testing for Lyme disease is considered "confusing and controversial." 19 While those patients who present with an erythema migrans (EM) rash are considered "infected" without the need for a confirmatory blood test and prescribed antibiotic treatment, ${ }^{20}$ those who do not have a rash but have a history of exposure or symptoms consistent with the diagnosis are instructed to undergo two-tiered testing: an ELISA first and then, if positive or indeterminate, a Western blot. ${ }^{21}$

This method of two-tiered testing recommended by the $\mathrm{CDC}$ is an indirect method of testing for the Borrelia bacteria and is considered by clinicians and researchers to have serious problems. ${ }^{22}$ The primary criticism is that the tests focus on diagnostic criteria that require antibodies to develop. Since these take time to develop, there is a high rate of false negative outcomes in early Lyme. ${ }^{22,23}$ Once a person is infected, antibodies may take many weeks to be produced. Waiting for evidence of their presence can mean that a window of early treatment is missed when the disease is potentially curable. A validated symptom questionnaire would help diagnose early acute tick-borne illness when antibody testing maybe negative.

The recommended two-tiered testing also misses as much as $44 \%$ of those with late Lyme disease. ${ }^{24}$ Part of the difficulty in finding antibodies against Borrelia is that they can be bound in circulating immune complexes. These tests also do not detect other related Borrelia species that cause Lyme-like illness, such as Borrelia miyamotoi and Borrelia sensu lato..$^{25-27}$

Scientific studies have also shown high rates of inter-and intralaboratory variabilities. Indirect methods such as the ELISA and the Western blot require subjective interpretation of results, leading to significant variability across and even 
within laboratories. ${ }^{28}$ For example, Ang et al ${ }^{28}$ compared a series of ELISA test kits for the diagnosis of neurological Lyme. Sensitivity varied from 20.9 to $97.7 \%$ for the kits. Direct testing methods of the infection also have proven challenging due to low bacterial counts that disperse quickly throughout the human body and the insufficient sensitivity of current detection methods. ${ }^{29}$

One of these direct methods, the polymerase chain reaction (PCR), can detect DNA from the Borrelia bacteria in fluid drawn from sites such as an infected joint (using synovial fluid) but is not highly sensitive in all other specimens. It can miss important areas such as a central nervous system infection, where it is much more difficult to detect ${ }^{30,31}$ and cannot reliably be used to track treatment response following antibiotics.

Newer, novel detection approaches to the detection of early Lyme disease are being developed to try and prevent longterm disability, including evaluating levels of cytokines and chemokines (serum inflammatory mediators). ${ }^{32}$ Many of these tests have not yet been through formal controlled clinical trials.

Late manifestations of Lyme disease are considered by many clinicians and by the CDC's own website as best diagnosed via patient "signs and symptoms"; in other words, Lyme is best conceived as a clinical diagnosis. ${ }^{33}$ Therefore, as a recent review concluded: "There is a need for the development and establishment of new clinical diagnostic tools with increased accuracy, sensitivity, and specificity...."34 A validated symptom questionnaire would be valuable in the diagnosis of chronic Lyme disease/posttreatment Lyme disease syndrome (PTLDS) where seronegativity is a wellestablished phenomenon. ${ }^{28,29,35}$

The consequences of insensitive blood tests leave many patients with long-standing "severe" or "very severe" health complaints. Some remain ill for $\geq 10$ years and wait $>2$ years for a diagnosis. ${ }^{36}$ The Ad Hoc International Lyme Disease Group and the Infectious Diseases Society of America (IDSA) make the assumption that patients' continuing symptoms of fatigue, joint and muscle pain, and cognitive dysfunction and disability are not the results of persistent, active infection. ${ }^{37,38}$ Other clinicians believe that there is persistence of Lyme and other tick-borne diseases ${ }^{39}$ and that these might be putting future generations at risk through maternal-fetal transmission ${ }^{40}$ and contaminated blood supplies. ${ }^{41}$

A validated screening questionnaire detecting Lyme disease in its initial stages when it is potentially curable, as well as in later stages, would benefit a rapidly growing number of individuals infected by Lyme. A standardized clinical questionnaire might provide physicians with such a tool, especially for patients experiencing symptoms but with seronegative laboratory results. This article examined whether a cluster of symptoms was more likely to be found in patients with Lyme disease than in non-Lyme/healthy patients and whether these symptoms provide a valid and reliable measure for clinicians. We tested whether the Horowitz Multiple Systemic Infectious Disease Syndrome (MSIDS) Questionnaire can be that tool.

\section{The Horowitz MSIDS Questionnaire (HMQ)}

Because numerous body systems are often concurrently affected by tick-borne disease, the third author (Horowitz), a long-time physician with a large Lyme disease patient population, in 2009 re-conceived tick-borne disease as part of an MSIDS. He developed protocols for his patients for simultaneously diagnosing and treating overlapping etiologic factors. ${ }^{42}$

In his 2013 book, Why Can't I Get Better...., ${ }^{43}$ he expanded a 2008 patient symptom questionnaire that was never formally validated. ${ }^{44}$ The expanded questionnaire, used to screen patients with Lyme disease, added new items, weights to various symptoms, items related to the likelihood of exposure to Lyme, and overall physical and mental health. Horowitz described "a gestalt of symptoms" that he found to be strongly associated with Lyme in his clinical practice. This was supported by evidence of Lyme-specific bands on Western blot blood test results. ${ }^{43}$ Although some of the symptoms on the questionnaire can be linked with other illnesses, there are specific symptoms that Horowitz found to be associated with Lyme disease, such as migratory joint pain, migratory muscle pain, and migratory nerve pain, which are not commonly found in other overlapping conditions, including fibromyalgia. ${ }^{45}$

Horowitz claimed that he could help the clinician reach an accurate assessment with a high "probability as to whether the patient may suffer from Lyme disease and associated tick-borne disorders" by examining this pattern of symptoms, exposure risk, and specific proteins associated with Borrelia species. Although the expanded questionnaire is in wide use in medical settings and online and has been reproduced across a variety of venues, it has never been empirically validated. Validation of the HMQ forms the basis of this research study. The questionnaire is described in detail in the "Methods" section.

\section{Study aims}

The study reported here had four aims. The first aim was to determine those clinical symptoms that serve as hallmarks of Lyme disease by conducting a factor analysis of the HMQ 
Symptom Checklist using a patient sample. Our second aim was to determine the psychometric properties of the HMQ, including its reliability and construct validity. Our third aim was to determine whether the HMQ score would show divergent validity, and our hypothesis was that the HMQ score would not correlate with personality factors on the Big Five. ${ }^{46}$ Our fourth aim was to determine whether scores on the HMQ would be able to discriminate self-identified healthy individuals from those with Lyme disease.

In addition, we examined the perceived toll of selfreported Lyme disease on a number of outdoor physical activities. Our hypothesis was that individuals with selfreported Lyme would report engaging in significantly fewer physical activities than the self-identified "healthy" participants, reflecting the level of disability demonstrated in prior NIH studies. ${ }^{47}$

\section{Strategy for data collection}

Three studies were conducted to address the study aims. Study 1 examined the psychometric properties of the HMQ in a sample of patients being treated for Lyme disease. The purpose was to factor analyze patients' clinical symptoms from the HMQ Symptom Checklist and to examine the internal reliability of that checklist.

Study 2 was designed to assess convergent validity by examining the correlations among key components of the questionnaire and by demonstrating that the components of the HMQ could discriminate between healthy individuals and those who self-identified as currently having Lyme disease. The study also sought to establish the divergent validity of the scale. In addition, we examined differences in outdoor activity between self-identified healthy individuals and those who self-identified as having Lyme disease now.

Study 3 consisted of a subsample of patients from the larger patient sample in Study 1, all of whom had a history of EM rash and/or laboratory evidence of Lyme disease compared with a new sample of self-identified healthy individuals. The purpose of this study was to demonstrate that the HMQ could accurately distinguish between healthy individuals and those with Lyme.

\section{Institutional review board approval}

Two protocols were submitted and approved for this research. The medical record review was certified as exempt on June 18, 2014, based on the existing data exemption by the Human Research Ethics Board (its Institutional Review Board) of the State University of New York (SUNY) at New Paltz. The survey component was certified exempt as anonymous survey research on June 2, 2014, by the SUNY New Paltz's Human Research Ethics Board.

\section{Study I \\ Methods}

\section{Participants}

The sample for Study 1 was a patient sample of 537 individuals being treated for Lyme at one of three medical practices. Two of the practices were primary care providers and one specialized in Lyme treatment. All three practices were following peer review guidelines for the diagnosis and treatment of Lyme disease. ${ }^{39}$ Patients completed the HMQ as a part of their treatment. Although all three medical practices were situated in the Northeastern United States, the Lyme specialty practice treats patients from regions beyond that area. All identifying information was removed, data coded, and entered into a database for statistical analysis.

Approximately $75 \%$ of the sample was female, and the mean age was 47.5 years (refer to Table 1 for the demographic breakdown for all three studies).

\section{Measures}

$\mathrm{HMQ}$

The HMQ consists of four sections. Each section provides clinicians with diagnostic information about the patients' likelihood of having Lyme disease or other tick-borne illnesses. For the patient sample, the scale was part of their medical record. For the healthy participants, the same scale was used in an online survey.

Table I Demographic breakdowns of the samples

\begin{tabular}{|c|c|c|c|c|c|}
\hline & $\begin{array}{l}\text { Study I: } \\
\text { Lyme patients }\end{array}$ & $\begin{array}{l}\text { Study 2: } \\
\text { Healthy }\end{array}$ & $\begin{array}{l}\text { Study 2: } \\
\text { Self-identified Lyme }\end{array}$ & $\begin{array}{l}\text { Study 3: } \\
\text { Healthy }\end{array}$ & $\begin{array}{l}\text { Study 3: } \\
\text { Verified Lyme }\end{array}$ \\
\hline \multicolumn{6}{|l|}{ Gender (\%) } \\
\hline Female & 72.8 & 81.1 & 84.5 & 76.9 & 80.1 \\
\hline Male & 27.2 & 17.9 & 14.7 & 22.9 & 19.9 \\
\hline Others & & 1.0 & 0.7 & 0.1 & \\
\hline Mean age (years) & 45.26 & 53.20 & 50.88 & 49.35 & 47.46 \\
\hline $\mathrm{N}$ & 537 & 217 & 782 & 568 & 236 \\
\hline
\end{tabular}


Section 1 of the HMQ contains 38 symptoms that are rated from 0 (indicating none) to 3 (indicating severe or extremely frequent symptoms). The response scale ranged from 0 (never), 1 (sometimes), 2 (most of the time), and 3 (all of the time).

Throughout this article, this section of the scale is referred to as the HMQ Symptom Checklist. It contains a range of symptoms, including fatigue, joint/muscle pain, and memory/ concentration problems, most likely to be seen in clinical practice with Lyme patients. This is scored by totaling the points across all items. Total scores can range from 0 to 114 .

Section 2 of the questionnaire is called the Lyme Incidence scale and contains items related to the likelihood of having Lyme. These questions include "Have you ever had a tick bite?"; "Have you ever had a bull's eye rash with flulike symptoms?"; "Do you live in a Lyme endemic area?"; and "Have you received a prior diagnosis of a non-specific autoimmune disorder?". This section also contains items about migratory symptoms. There are 10 items in this section and each is worth a varying number of points. For example, a tick bite with a rash followed by flulike symptoms is worth five points. Scores on this section can range from 0 to 34 .

Section 3 comes from two of the four questions from the CDC's own Healthy Days Core Module ${ }^{48}$ in which patients are asked about their overall health (physical and mental health) over the last 30 days. Points are awarded as follows: $0-5$ days, one point; 6-12 days, two points; 13-20 days, three points; 21-30 days, four points. For the two questions, combined scores can range from 2 to 8 .

Section 4 creates a score for common Lyme symptoms. This is referred to as the Common Lyme score. The Common Lyme score was developed by Horowitz, based on research by Shadick et al, ${ }^{49}$ to create a score reflecting the symptoms that Horowitz observed in his clinical practice linked to a higher probability of having Lyme disease. Five additional points are awarded if the following symptoms are rated as severe or extremely frequent: 1) fatigue, 2) forgetfulness and poor short-term memory, 3) joint pain or swelling, 4) tingling, numbness, burning, or stabbing sensations, and 5) disturbed sleep: too much, too little, and early awakening. In the original version of the scale, this is scored as 0 or 5 . If all five of these symptoms are severe or extremely frequent, the Common Lyme score would be 5. If not, the Common Lyme score would be 0 . For the purpose of this article, however, the Common Lyme score was calculated as an interval level variable and a point was given for each symptom that was rated severe or extremely frequent. Using this scoring, the Common Lyme score ranged from 0 to 5 . The purpose of this change was so that this score could be analyzed using parametric statistics such as analysis of variance (ANOVA) and Pearson product-moment correlations.

An overall HMQ score is calculated by adding the points for the four sections together.

\section{Results}

\section{Factor analysis}

SPSS was used to conduct an exploratory factor analysis of the HMQ Symptom Checklist to examine its factor structure and construct validity. The HMQ Symptom Checklist items were factor analyzed using maximum likelihood estimation. An oblique rotation was used to identify factors. Because symptoms often co-occur, this allowed correlations among the factors.

We considered an item to load on a factor if it had a $\geq 0.25$ loading. In cases where an item loaded on more than one factor at the level of $\geq 0.25$, we considered it to load on the factor with its highest loading. In all cases, the primary loading made the most sense in terms of the factors and latent variables underlying the factors.

Six factors were identified by examining the scree plot, the Eigenvalues, and variance explained. As factor analysis involves both statistical analysis and judgment, the factor labels are based on interpretations. These were labeled neuropathy, cognitive dysfunction, musculoskeletal pain, fatigue, dysautonomia, and cardio/respiratory. The items and their factor loadings are listed in Table 2.

The first four factors appear to correspond with common Lyme symptoms identified by Horowitz ${ }^{43}$ and others ${ }^{49}$ and reflected in Section 4 of the HMQ. These include the following five items:

- Tingling, numbness, burning, or stabbing sensations

- Forgetfulness and poor short-term memory

- Joint pain or swelling

- Fatigue

- Disturbed sleep: too much, too little, and early awakening

Tingling, numbness, burning, or stabbing sensations loaded on the neuropathy factor. Forgetfulness, poor shortterm memory loaded on the cognitive dysfunction factor. Joint pain or swelling loaded on the musculoskeletal pain factor. Fatigue and disturbed sleep: too much, too little, and early awakening loaded on the fatigue factor. These loadings appear to support the construct validity of the checklist. Items representing the hallmarks of Lyme disease correlated together to form cohesive factors.

The two additional factors, dysautonomia and cardio/ respiratory, reflect typical symptoms of inflammation and 
Table 2 Study I - factor analysis using maximum likelihood estimation and oblique rotation

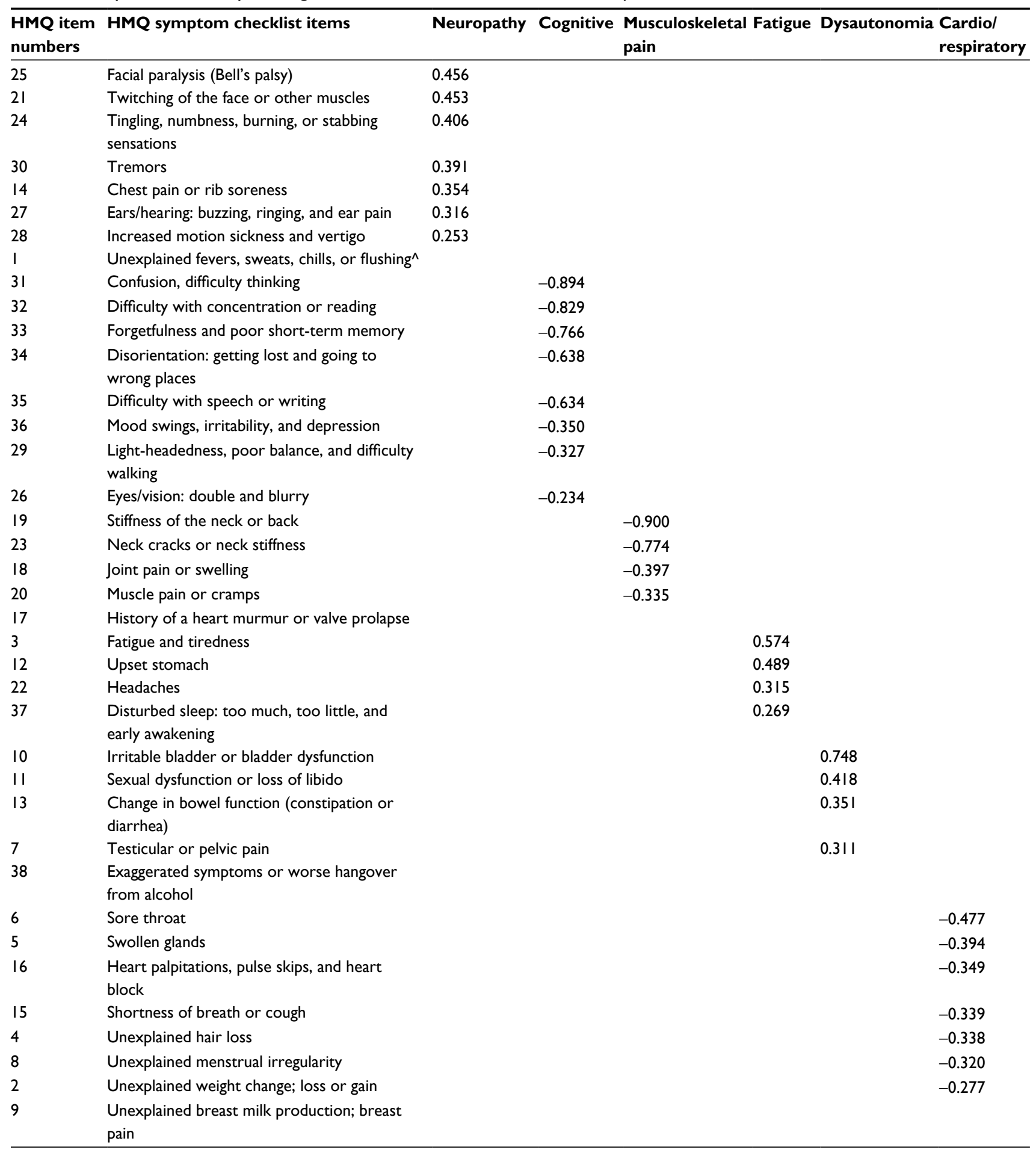

Note: ^Item failed to load on any factor at the 0.25 level or higher.

illness that are not limited to Lyme disease or other tick-borne illnesses but when combined with symptoms from the other factors can be indicative of Lyme disease. If fever, sweats, chills, flushing, an unexplained cough, and/or shortness of breath are present, these symptoms may reflect a possible co-infection like babesiosis (a malaria-like parasite).

\section{Reliability}

To demonstrate reliability and internal consistency, we used Cronbach's coefficient alpha. ${ }^{50}$ Table 3 presents the Cronbach's coefficient alphas for the HMQ (ie, the scores from the sections of the scale), HMQ Symptom Checklist, the Lyme Incidence score, the Common Lyme score, and all of the 
Table 3 Study I - reliability using Cronbach's coefficient alpha based on standardized items

\begin{tabular}{ll}
\hline HMQ sections and factors & Cronbach's alpha \\
\hline Overall HMQ scale & 0.75 \\
HMQ Symptom Checklist & 0.93 \\
Common Lyme score & 0.66 \\
Lyme Incidence & 0.55 \\
Neuropathy & 0.73 \\
Cognitive & 0.87 \\
Musculoskeletal pain & 0.80 \\
Fatigue & 0.70 \\
Dysautonomia & 0.61 \\
Cardio/respiratory & 0.72 \\
\hline
\end{tabular}

Abbreviation: HMQ, Horowitz Multiple Systemic Infectious Disease Syndrome Questionnaire.

factors from the HMQ Symptom Checklist. The HMQ Symptom Checklist and all of the factors of this scale demonstrated acceptable levels of reliability. ${ }^{51}$ The reliability for the HMQ Symptom Checklist was 0.93 . The Lyme Incidence scale had the lowest reliability $(0.55)$. In examining this scale, there are several possible reasons for this result. ${ }^{52}$ First, this scale contains two items that ask whether an individual has had a tick bite with a rash and a tick bite without a rash. Typically, individuals who answer one affirmatively do not answer the second affirmatively. In fact, if the tick bite without a rash question is dropped from the scale, the Cronbach's alpha would increase to 0.59 . Second, some individuals indicated that they had trouble answering the question: "Have you had a tick bite with an EM (bull's eye rash), or an undefined rash, followed by flulike symptoms?" Some indicated that they did have a tick bite with a rash, but they did not have flulike symptoms immediately following the rash. So they indicated a negative answer for this question. The compound nature of this question could cause some inconsistency in how it is answered. Some refinement of these items would likely enhance the reliability of this scale. Third, the scale includes two distinctly different sets of items. One set focuses on the likelihood that a person received a tick bite from a tick infected with the bacteria that causes Lyme disease, while the other focuses on migratory symptoms. We look more closely at these migratory symptoms in Studies 2 and 3.

\section{Study 2 \\ Methods}

\section{Participants}

Study 2 participants were recruited through email, social media, and snowball sampling. Invitations to participate were posted to both the web pages and social media pages of Lyme groups such as the Bay Area Lyme Foundation, Lyme Disease
Association, and Lyme Action Network. We also recruited participants from non-Lyme associated groups including outdoor activity groups - such as hiking and biking groups (eg, the Mid-Hudson Adirondack Mountain Club and Hudson Valley Hikers) and a number of national hiking and outdoor recreation groups. Potential participants were sent an email invitation containing a link to the online survey. After clicking the link, the participants viewed a page that explained the purpose of the survey and provided information necessary for them to make an informed decision about whether to participate or not. All survey responses were anonymous. No identifying information was requested, and IP addresses were not collected.

Study 2 compared responses for individuals who selfidentified as having Lyme or as healthy. Participants were categorized based on a survey item that asked if the individual was "suffering from Lyme now." Individuals responding "yes" were classified as individuals with self-identified Lyme. Those responding "no" were classified as healthy, if they also responded yes to being in good health. Of the 999 survey participants, 782 participants indicated that they were suffering from Lyme now and 217 participants indicated that they were healthy.

To assess whether our operational definition of "selfidentified Lyme" was valid, we compared the Lyme categorization to the following two criteria that indicate whether a person has Lyme disease or not based on the CDC 2011 Surveillance Criteria: ${ }^{53}$ 1) positive Lyme testing and 2) a tick bite with a bull's eye rash. Using these two items, we classified participants into the following two groups: CDC Lyme and CDC non-Lyme. We compared the two categorization strategies for overlap.

Using the two items from the Lyme Incidence scale, we found that $84.1 \%$ of the individuals in the self-identified Lyme group met one or both of these CDC Lyme surveillance criteria. ${ }^{53}$ Only $30 \%$ of the healthy group met these criteria. Using discriminant analysis, these elements of the CDC Surveillance Criteria accurately predicted the correct Lyme classification for $81.1 \%$ of the survey respondents (ie, whether they self-identified as healthy or as having Lyme). Thus, this analysis confirmed that this study's operational definition of Lyme status was valid.

\section{Measures}

HMQ

The HMQ, as described earlier in Study 1, was administered with two minor modifications to Section 1 of the scale (the HMQ Symptom Checklist). Study 2 used the scale anchors 
that were published in Horowitz's 2013 book. ${ }^{43}$ The response scale was 0 (none), 1 (mild), 2 (moderate), and 3 (severe). Since this version of the scale was the most widely distributed, we wanted to validate it using the published anchors.

In addition, a "not applicable" option was added. This option allowed the participants to indicate whether they did not experience the symptom or the symptom was not applicable to them. For example, almost all male respondents reported the item "unexplained menstrual irregularity" was not applicable to them. When scoring the checklist, not applicable responses were coded as 0 and were primarily used to examine the utility of the items on the scale.

\section{Big Five personality characteristics}

The Big Five personality characteristics (openness, conscientiousness, extraversion, agreeableness, and neuroticism) were measured by the Big Five Inventory short form (BFI-s) developed for the German Socio-Economic Panel Study. This measure of the Big Five personality characteristics was shown to have acceptable levels of both reliability and validity. ${ }^{46}$ The rationale for including this measure was to show discriminant validity with the HMQ. We predicted that the HMQ would not correlate with these five characteristics of personality.

\section{Outdoor activity survey}

The outdoor activity survey was a 13-item measure that was designed for this study. Items on the survey were developed based on the National Survey on Recreation and the Environment and the Oregon Outdoor Recreation Survey. ${ }^{54}$ The survey measured the frequency that an individual engaged in common outdoor activities, such as hiking, biking, swimming, picnicking, and horseback riding. Participants rated the frequency that they engaged in these activities from 0 (never) to 4 (very often). Scores on this scale ranged from 0 to 52 .

\section{Results}

\section{Convergent and divergent validity}

We examined the convergent and divergent validity of the HMQ. For convergent validity, we expected that scores on the sections of the HMQ and its factors would correlate highly with each other. For divergent validity, we predicted that scores on the sections of the HMQ would correlate less highly with the Big Five personality factors (conscientiousness, agreeableness, neuroticism, openness, and extraversion). To analyze convergent and divergent validity, we calculated Pearson productmoment correlation coefficients using SPSS version 24.

Table 4 shows the multitrait matrix and indicates that these predictions were supported by the data. The HMQ score,

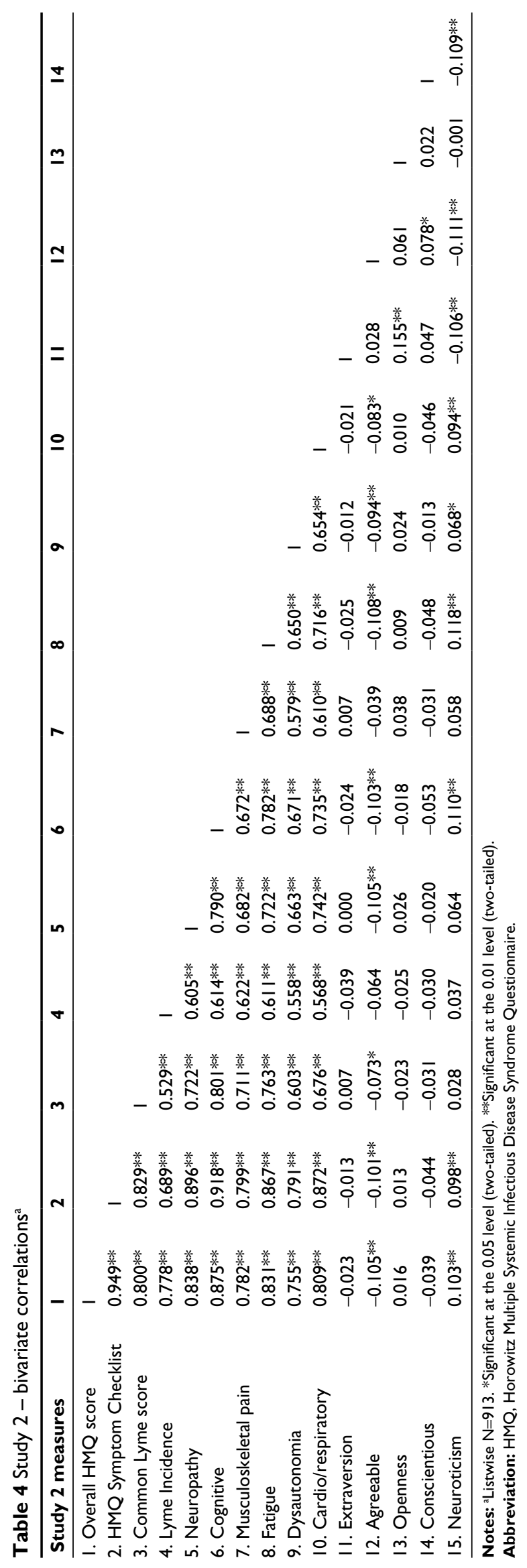


scores on each of its sections, and the symptom factor scores correlated highly with each other. The correlations ranged from 0.53 to 0.95 and were all significant at the $P<0.001$ level.

In contrast, the HMQ and its factors did not significantly correlate with conscientiousness, openness, and extraversion. In fact, these correlations were near zero (ranging from 0.00 to 0.05 ). Agreeableness was found to have small negative correlations with the HMQ score, its sections, and factors. While some of these correlations may have been significant, they ranged from -0.04 to -0.11 and accounted for $1 \%$ of the variance or less.

The questionnaire scores and factors also showed significant but small positive correlations with neuroticism. These ranged from 0.03 to 0.12 . The positive correlations are consistent with what is known about the neuroticism construct. ${ }^{55}$ Recent research has shown that neuroticism scores change with life experiences. Negative life experiences such as coping with chronic illness lead to increased levels of neuroticism. ${ }^{56}$ This suggests that we should expect a small positive correlation between HMQ scores (ie, Lyme symptoms) and neuroticism. Nevertheless, these correlations, however, do not account for much of the variance. The largest accounts for $\sim 1 \%$ of the variance.

\section{Predicting Lyme status - one-way ANOVA}

We examined whether Lyme status predicted HMQ scores. We used SPSS version 24 to calculate a one-way ANOVA for the overall HMQ scores comparing the self-identified Lyme group with the healthy group. The ANOVA showed a significant effect $(F[1,891]=844.87, P<0.001)$, indicating that HMQ scores significantly differed across the two groups: self-identified Lyme $(M=88.66)$ and healthy $(M=28.50)$. We calculated the effect size using eta ${ }^{2} .^{57}$ The overall eta ${ }^{2}$ was 0.49. According to Cohen, ${ }^{58}$ this is a large effect size and indicates that $\sim 49 \%$ of the variance in HMQ scores could be explained by Lyme group.

The same pattern of findings was found for HMQ Symptom Checklist scores, Common Lyme score, Lyme Incidence, and all of the HMQ factor scores (Table 5.) The effect sizes ranged from 0.243 to 0.454 , suggesting that Lyme group explained $\sim 24-45 \%$ of the variance in these scores.

In contrast, the ANOVA results for only one of the Big Five factors were significant: agreeableness (Table 6). These results indicated that the self-identified Lyme group reported slightly lower levels of agreeableness than the healthy group. The effect sizes, however, were extremely small ${ }^{58}$ and accounted for $<1 \%$ of the variance.

To test the sensitivity of the data, ${ }^{59}$ we examined whether the data for all of the variables met the normality assumption for ANOVA and found that responses of healthy individuals were skewed toward the zero end of the scales. For instance, healthy individuals were likely to report that they never experienced any of the symptoms on the HMQ Symptom Checklist. ANOVA is robust to deviations from normal

Table 5 Study 2 - mean and one-way analysis of variance results for the HMQ

\begin{tabular}{|c|c|c|c|c|c|c|c|c|c|}
\hline \multirow{2}{*}{$\begin{array}{l}\text { HMQ sections and } \\
\text { factors }\end{array}$} & \multicolumn{2}{|c|}{ Healthy } & \multicolumn{2}{|c|}{ Self-identified Lyme } & \multirow{2}{*}{$d f_{I}$} & \multirow{2}{*}{$\mathrm{df}_{2}$} & \multirow[t]{2}{*}{$\boldsymbol{F}$} & \multirow[t]{2}{*}{ Significance } & \multirow[t]{2}{*}{$\mathrm{Eta}^{2}$} \\
\hline & $M$ & SD & $\mathbf{M}$ & SD & & & & & \\
\hline Overall HMQ score & 28.50 & 18.18 & 88.66 & 27.52 & I & 891 & 844.87 & $<0.001$ & 0.487 \\
\hline HMQ Symptom Checklist & 16.12 & 11.54 & 55.60 & 20.34 & 1 & 997 & 750.162 & $<0.001$ & 0.429 \\
\hline Lyme Incidence & 10.00 & 8.02 & 23.57 & 5.55 & I & 922 & 766.77 & $<0.001$ & 0.454 \\
\hline Common Lyme score & 0.25 & 0.63 & 2.41 & 1.56 & 1 & 997 & 393.82 & $<0.001$ & 0.283 \\
\hline Neuropathy & 0.28 & 0.31 & 1.24 & 0.65 & I & 997 & 442.70 & $<0.001$ & 0.307 \\
\hline Cognitive & 0.35 & 0.38 & 1.64 & 0.75 & I & 997 & 603.00 & $<0.001$ & 0.377 \\
\hline Musculoskeletal pain & 0.89 & 0.70 & 2.13 & 0.78 & 1 & 997 & 449.79 & $<0.001$ & 0.311 \\
\hline Fatigue & 0.86 & 0.58 & 2.07 & 0.64 & I & 997 & 635.42 & $<0.001$ & 0.389 \\
\hline Dysautonomia & 0.38 & 0.47 & 1.33 & 0.74 & I & 997 & 320.48 & $<0.001$ & 0.243 \\
\hline Cardio/respiratory & 0.27 & 0.33 & 1.17 & 0.63 & I & 997 & 410.07 & $<0.001$ & 0.291 \\
\hline
\end{tabular}

Abbreviation: HMQ, Horowitz Multiple Systemic Infectious Disease Syndrome Questionnaire.

Table 6 Study 2 - mean and one-way analysis of variance results for the Big Five personality characteristics

\begin{tabular}{|c|c|c|c|c|c|c|c|c|c|}
\hline \multirow{2}{*}{$\begin{array}{l}\text { Big } 5 \text { personality } \\
\text { factor }\end{array}$} & \multicolumn{2}{|c|}{ Healthy } & \multicolumn{2}{|c|}{ Self-identified Lyme } & \multirow[t]{2}{*}{$\mathbf{d f}_{\mathbf{I}}$} & \multirow[t]{2}{*}{$\mathbf{d f}_{2}$} & \multirow[t]{2}{*}{$\boldsymbol{F}$} & \multirow[t]{2}{*}{ Significance } & \multirow[t]{2}{*}{$\mathrm{Eta}^{2}$} \\
\hline & $M$ & SD & M & SD & & & & & \\
\hline Extraversion & 4.41 & 0.89 & 4.34 & 0.97 & 1 & 956 & 0.79 & 0.375 & 0.001 \\
\hline Agreeable & 4.96 & 0.97 & 4.79 & 0.98 & 1 & 959 & 4.38 & 0.037 & 0.005 \\
\hline Openness & 4.76 & 0.87 & 4.70 & 0.86 & 1 & 952 & 0.96 & 0.328 & 0.001 \\
\hline Conscientious & 4.92 & 0.81 & 4.81 & 1.01 & 1 & 959 & 2.00 & 0.157 & 0.002 \\
\hline Neuroticism & 4.16 & 1.10 & 4.28 & 1.17 & 1 & 957 & 1.65 & 0.199 & 0.002 \\
\hline
\end{tabular}


distributions. ${ }^{60-62}$ To verify our results, however, we examined whether the same pattern of results would hold when tested with a nonparametric test of ranks, the Kruskal-Wallis test. ${ }^{63}$ This nonparametric test compares differences among groups but does not make the assumption that the underlying distributions are normal. The Kruskal-Wallis results exactly paralleled the primary ANOVA results with no exceptions and demonstrated the robustness of our ANOVA findings (refer to Table 7 for the Kruskal-Wallis analyses).

\section{Predicting Lyme status - discriminant analysis}

We conducted a discriminant analysis using SPSS to test whether the HMQ and its component scores could accurately classify individuals into Lyme status, ie, the self-identified Lyme and healthy categories. Discriminant analysis is used when the goal is to assess whether a set of variables (like the components of the HMQ) can predict category membership (in this case Lyme status). ${ }^{64}$ We also conducted a discriminant analysis using the Big Five components to predict Lyme status. Table 8 shows the results for all four discriminant analyses and the percentages of people correctly classified into the healthy and self-identified Lyme groups for all four discriminant analyses.

The discriminant analysis for the overall HMQ score showed that HMQ score accurately predicted the Lyme category for $89.1 \%$ of the respondents. Accuracy for the Healthy group was $91.5 \%$ and accuracy for the self-identified Lyme group was $88.5 \%$.

A second discriminant analysis was conducted examining the sections of the HMQ (the HMQ Symptom Checklist, the Lyme Incidence score, the Healthy Days score, and the Common Lyme score). Taken together, the sections of the HMQ accurately predicted Lyme category. The overall accuracy was $89.5 \%$. The HMQ factor scores showed an $85.6 \%$ accuracy rate. These factors were then entered using a stepwise procedure. In the first step, the model selected the factor with the best categorization accuracy and proceeded to enter additional factors in subsequent steps until the remaining factors did not add significantly to the categorization accuracy. The factor entered in the first step was the fatigue factor. The cognitive dysfunction factor was entered in the second step, and the musculoskeletal pain factor was entered in the third

Table 7 Kruskal Wallis Results for the Horowitz MSIDS Questionnaire (HMQ) and the Big Five Personality Characteristics for Study 2 and 3

\begin{tabular}{|c|c|c|c|c|c|c|c|}
\hline \multirow{2}{*}{$\begin{array}{l}\text { Study } 2 \\
\text { HMQ Sections and Factors }\end{array}$} & \multicolumn{2}{|l|}{ Healthy } & \multicolumn{2}{|c|}{ Self-Identified Lyme } & \multirow[t]{2}{*}{ df } & \multirow[t]{2}{*}{ Chi-Square } & \multirow[t]{2}{*}{ Significance } \\
\hline & Mean Rank & $\mathbf{N}^{\mathbf{a}}$ & Mean Rank & $\mathbf{N}^{\mathbf{a}}$ & & & \\
\hline Overall HMQ Score & 123.96 & 200 & 540.74 & 694 & $\mathrm{I}$ & 404.52 & $<0.001$ \\
\hline HMQ Symptom Checklist & 144.58 & 218 & 599.72 & 782 & I & 423.42 & $<0.001$ \\
\hline Lyme Incidence & 176.86 & 205 & 544.47 & 720 & $\mathrm{I}$ & 302.82 & $<0.001$ \\
\hline Common Lyme Score & 196.49 & 218 & 585.25 & 782 & I & 301.71 & $<0.001$ \\
\hline Neuropathy & 174.50 & 218 & 591.38 & 782 & I & 356.54 & $<0.001$ \\
\hline Cognitive & 161.06 & 218 & 595.13 & 782 & I & 385.93 & $<0.001$ \\
\hline Musculo- skeletal Pain & 211.08 & 218 & 581.18 & 782 & I & 282.92 & $<0.001$ \\
\hline Fatigue & 180.50 & 218 & 589.71 & 782 & $\mathrm{I}$ & 345.31 & $<0.001$ \\
\hline Dysautonomia & 220.88 & 218 & 578.45 & 782 & 1 & 263.78 & $<0.001$ \\
\hline Cardio/ Respiratory & 181.06 & 218 & 589.55 & 782 & 1 & 342.52 & $<0.001$ \\
\hline Extraversion & 492.99 & 204 & 476.49 & 755 & $\mathrm{I}$ & 0.58 & 0.448 \\
\hline Agreeableness & 519.10 & 206 & 471.25 & 756 & $\mathrm{I}$ & 4.86 & 0.027 \\
\hline Openness & 497.36 & 206 & 472.68 & 749 & I & 1.32 & 0.250 \\
\hline Conscientious & 491.87 & 204 & 478.71 & 758 & I & 0.37 & 0.545 \\
\hline Neuroticism & 455.74 & 204 & 487.18 & 756 & $\mathrm{I}$ & 2.08 & 0.149 \\
\hline Study 3 & \multicolumn{2}{|l|}{ Healthy } & \multicolumn{2}{|c|}{ Verified Lyme } & df & Chi-Square & Significance \\
\hline HMQ Sections and Factors & Mean Rank & $\mathbf{N}^{\mathbf{a}}$ & Mean Rank & $\mathbf{N}^{\mathbf{a}}$ & & & \\
\hline Overall HMQ Score & 265.32 & 499 & 574.08 & 219 & $\mathrm{I}$ & 337.42 & $<0.001$ \\
\hline HMQ Symptom Checklist & 317.10 & 568 & 607.22 & 235 & $\mathrm{I}$ & 260.24 & $<0.001$ \\
\hline Lyme Incidence & 276.63 & 527 & 615.44 & 233 & I & 387.14 & $<0.001$ \\
\hline Common Lyme Score & 339.73 & 568 & 553.58 & 236 & I & 253.76 & $<0.001$ \\
\hline Neuropathy & 333.86 & 568 & 567.70 & 236 & I & 175.36 & $<0.001$ \\
\hline Cognitive & 323.57 & 568 & 592.47 & 236 & I & 227.54 & $<0.001$ \\
\hline Musculo- skeletal Pain & 326.82 & 568 & 584.65 & 236 & 1 & 208.58 & $<0.001$ \\
\hline Fatigue & 332.33 & 568 & 571.39 & 236 & I & 179.64 & $<0.001$ \\
\hline Dysautonomia & 362.77 & 568 & 496.81 & 235 & I & 58.18 & $<0.001$ \\
\hline Cardio/ Respiratory & 344.22 & 568 & 542.77 & 236 & I & 125.89 & $<0.001$ \\
\hline
\end{tabular}

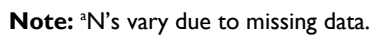

Abbreviation: HMQ, Horowitz Multiple Systemic Infectious Disease Syndrome Questionnaire. 
Table 8 Study 2 - discriminant analysis results

\begin{tabular}{llllllll}
\hline Test of function(s) & $\begin{array}{l}\text { Wilks' } \\
\text { lambda }\end{array}$ & $\begin{array}{l}\text { Chi } \\
\text { square }\end{array}$ & & df & Significance & \multicolumn{2}{l}{$\begin{array}{l}\text { Percent correctly classified by discriminant } \\
\text { analysis }\end{array}$} \\
\cline { 3 - 7 } & & & & & Overall & Healthy & Self-identified Lyme \\
\hline Overall HMQ score & 0.513 & 593.89 & $\mathrm{I}$ & $<0.001$ & 89.1 & 91.5 & 88.5 \\
HMQ sections & 0.458 & 693.43 & 4 & $<0.001$ & 89.5 & 84.4 & 90.9 \\
HMQ factors & 0.547 & 599.12 & 6 & $<0.001$ & 85.6 & 89.9 & 84.4 \\
Big Five & 0.992 & 7.73 & 5 & 0.171 & 51.7 & 52.8 & 51.4 \\
\hline
\end{tabular}

Abbreviation: HMQ, Horowitz Multiple Systemic Infectious Disease Syndrome Questionnaire.

step. The other three factors (neuropathy, dysautonomia, and cardio/respiratory) did not contribute additional significant variance to the prediction. Overall, these three factors led to $85.0 \%$ of the sample being correctly categorized into the self-identified Lyme and the healthy groups.

In comparison, the Big Five factors led to much worse categorization overall. The discriminant function when all five personality factors were entered into the model at the same time did not reach traditional levels of statistical significance. The overall accuracy of the model was $51.7 \%$. This is very close to the classification that you would expect by chance alone.

The HMQ score, its components, and its factors accurately discriminated healthy individuals from those who self-identified as suffering from Lyme. The Big Five factors performed only slightly better than chance. These results demonstrated the discriminant validity of the HMQ.

\section{Outdoor activity survey}

The frequency of outdoor activity significantly varied by Lyme status $(F[1,928]=12.96, P<0.001)$. The self-identified Lyme group $(M=24.69, \mathrm{SD}=7.49)$ reported engaging in significantly fewer outdoor activities than the healthy group $(M=26.71, \mathrm{SD}=5.40)$.

\section{Additional analyses and item issues}

Initial analysis of the 38 items on the HMQ Symptom Checklist revealed that responses to four items showed a high number of not applicable responses. These items showed a dramatically higher rate of not applicable responses than other items on the scale. In addition, the items "unexplained menstrual irregularity" and "unexplained breast milk production; breast pain" were primarily rated as not applicable by males. In fact, $\sim 1 / 3$ of the males taking the online survey responded with not applicable to these two items. Item 38 focused on hangover symptoms. It is likely that nondrinkers felt that this symptom was irrelevant to them.

The Lyme Incidence scale had lower internal reliability in Study 1. In examining the questions on this scale, there appears to be two types of questions. One type focuses on whether a person has had a tick bite, a rash, lives in a Lyme endemic area, and has a family member with Lyme. These items assess the likelihood that a person was exposed to a bite by a tick carrying Lyme disease.

The other set of items focuses on migratory symptoms:

- You experience migratory muscle pain.

- You experience migratory joint pain.

- You experience tingling/burning/numbness that migrates and/or comes and goes.

To look at whether any migratory symptom predicted patient vs healthy status, we created a "migratory" variable that was scored 1 if the response to any of the three items was yes and 0 if all three responses were no. A chi square analysis showed that migratory symptoms predicted patient status $\left(\chi^{2}[1]=338.48, P<0.001\right)$. Of the self-identified Lyme group, $94.2 \%$ indicated that they experienced at least one of the migratory symptoms, while only $41 \%$ of the healthy individuals did.

Similarly, we compared patient status with whether an individual experienced 1, 2, or 3 migratory symptoms. Again, the chi square analysis showed a significant relationship between patient status and migratory symptoms $\left(\chi^{2}\right.$ $[3]=444.92, P<0.001)$. Self-identified Lyme respondents reported a much higher frequency of migratory symptoms than did healthy participants. Approximately $79.3 \%$ of those who self-identified as having Lyme reported experiencing all three types of migratory pain, while only $16.5 \%$ of the healthy individuals reported experiencing all three types of migratory pain.

\section{Study 3}

Study 3 was conducted to address several concerns and limitations in Study 2. In Study 2, Lyme status was based on self-identification. Although the results indicated that this self-identification was consistent with two markers related to elements of the CDC Surveillance Criteria $^{53}$ used to determine whether an individual has Lyme disease or 
not (ie, tick bite with an EM rash or a positive Lyme test), Lyme disease status was not verified. Study 3 was designed to compare individuals with a confirmed diagnosis of Lyme disease with self-identified healthy individuals. For this study, we defined a healthy respondent as one who had never been diagnosed with Lyme disease and who responded yes to a survey question about being in good health. These criteria exclude individuals who may have had a Lyme diagnosis in the past, received treatment, and were now feeling better. Healthy respondents were those who indicated that they had never sought or received treatment for Lyme disease.

A second issue addressed by Study 3 focused on the scale used for the HMQ Symptom Checklist. Study 2 used the scale that was published in Horowitz's 2013 book. ${ }^{43}$ In Study 2, the 38 symptoms on the checklist were rated in terms of severity (from 0 indicating none to 3 indicating severe). Patients who received this survey from their health care provider rated the symptoms in terms of frequency with 0 indicating never and 3 indicating all of the time. For a more precise comparison, Study 3 used the same scale that was given to the patients. Thus, all respondents answered the HMQ Symptom Checklist using the following scale: $0=$ never, $1=$ sometimes, $2=$ most of the time, and $3=$ all of the time.

\section{Methods}

\section{Participants}

Study 3 examined a subsample of the patients from Study $1(\mathrm{~N}=236)$ and gathered additional data from a new group of 568 self-identified healthy individuals using an online survey. All 236 patient participants in Study 3 were new patients to the three practices and who had documented evidence of Lyme disease. These patients met the criteria for a clinical diagnosis of Lyme disease: 108 participants met the criterion by self-reporting a tick bite that was followed by an EM rash with positive Lyme laboratory testing including a positive immunofluorescent antibodies, PCR, and an IgG/ IgM Western blot, 73 participants met the criterion with just positive Lyme laboratory testing, and 55 participants met the criterion with just a tick bite followed by an EM rash.

The healthy sample was recruited using email, social media, and snowball sampling. We recruited participants from non-Lyme associated groups including outdoor activity groups, such as hiking and biking groups (eg, the Mid-Hudson Adirondack Mountain Club and Hudson Valley Hikers), and a number of national hiking and outdoor recreation groups. Participants were also recruited from the social media contacts of the authors. Potential participants were sent an email invitation containing a link to the online survey. After clicking the link, the participants viewed a page that explained the purpose of the survey and provided information necessary for them to make an informed decision about whether to participate or not. All survey responses were anonymous. No identifying information was requested, and IP addresses were not collected.

The Study 3 healthy participants consisted of 584 people who indicated that they had never had a Lyme diagnosis and who responded yes to the survey question, "Do you consider yourself to be in good health?" The first item on the survey asked whether or not the individual was ever diagnosed with Lyme disease. If individuals answered yes, then they were immediately sent to the end of the survey and thanked for their time. If individuals answered no, then they were allowed to complete the survey. In addition, individuals who answered no to the question "Do you consider yourself to be in good health?" were excluded from the healthy group. Of the 584 participants meeting these criteria, 16 participants indicated that they had received a positive Lyme test. Because this is an important criterion for determining who has had Lyme disease, these 16 individuals were excluded from analyses resulting in a sample size of 568 .

\section{Measures}

$\mathrm{HMQ}$

The HMQ, as described earlier in Study 1, was administered. As in Study 1, the response scale for Section 1 (the HMQ Symptom Checklist) ranged from 0 (never), 1 (sometimes), 2 (most of the time), and 3 (all of the time).

\section{Results}

\section{Predicting patient status - one-way ANOVA}

We conducted a one-way ANOVA using SPSS version 24 to examine whether patient status predicted HMQ scores. The ANOVA results showed that the overall HMQ score $(F[1,716]=770.820, P<0.001)$ differed across the following two groups: patient $(M=62.14)$ and healthy $(M=20.91)$. As in Study 2, eta ${ }^{2}$ was used to assess the strength of the effect. ${ }^{58}$ The overall eta $^{2}$ was 0.52 , indicating a large effect that accounted for $\sim 52 \%$ of the variance in the HMQ scores between patient vs healthy status.

The same pattern was found for HMQ Symptom Checklist, Lyme Incidence, Common Lyme score, and all of the HMQ factor scores (Table 9.) Using $\mathrm{eta}^{2}$, the effect sizes ranged from 0.10 to 0.62 , suggesting that Lyme group explained $\sim 10 \%$ (dysautonomia) to $62 \%$ (Lyme Incidence) of the variance in these scores. The relatively small amount of variance explained by dysautonomia was not surprising 
Table 9 Study 3-Means and One-Way Analysis of Variance Results for the Horowitz MSIDS Questionnaire

\begin{tabular}{|c|c|c|c|c|c|c|c|c|c|}
\hline \multirow[b]{2}{*}{ HMQ Sections and Factors } & \multicolumn{2}{|c|}{ Healthy } & \multicolumn{2}{|c|}{ Verified Lyme } & \multirow[t]{2}{*}{ df $_{\mathbf{I}}$} & \multirow[t]{2}{*}{$\mathbf{d f}_{2}$} & \multirow[t]{2}{*}{$\boldsymbol{F}$} & \multirow[t]{2}{*}{ Significance } & \multirow[t]{2}{*}{$\mathrm{Eta}^{2}$} \\
\hline & $\mathbf{M}$ & SD & $\mathbf{M}$ & SD & & & & & \\
\hline Overall HMQ Score & 20.91 & 14.75 & 62.14 & 24.61 & I & 716 & 770.82 & $<0.001$ & 0.52 \\
\hline HMQ Symptom Checklist & 13.95 & 11.19 & 35.20 & 17.80 & 1 & 801 & $4 \mid 4.24$ & $<0.001$ & 0.34 \\
\hline Common Lyme Score & 0.13 & 0.46 & 1.28 & 1.41 & 1 & 802 & 301.36 & $<0.001$ & 0.27 \\
\hline LYME Incidence Score & 4.50 & 4.43 & 20.39 & 7.84 & 1 & 758 & 1259.68 & $<0.001$ & 0.62 \\
\hline Neuropathy & 0.24 & 0.30 & 0.74 & 0.58 & 1 & 802 & 261.04 & $<0.001$ & 0.25 \\
\hline Cognitive & 0.33 & 0.36 & 1.06 & 0.69 & I & 802 & 387.06 & $<0.001$ & 0.33 \\
\hline Musculoskeletal Pain & 0.64 & 0.56 & 1.52 & 0.78 & I & 802 & 318.92 & $<0.001$ & 0.29 \\
\hline Fatigue & 0.79 & 0.49 & 1.50 & 0.68 & 1 & 802 & 274.60 & $<0.001$ & 0.26 \\
\hline Dysautonomia & 0.37 & 0.40 & 0.73 & 0.65 & 1 & 801 & 91.38 & $<0.001$ & 0.10 \\
\hline Cardio/Respiratory & 0.26 & 0.31 & 0.62 & 0.48 & I & 802 & 161.69 & $<0.001$ & 0.17 \\
\hline
\end{tabular}

Abbreviation: HMQ, Horowitz Multiple Systemic Infectious Disease Syndrome Questionnaire

Table 10 Study 3-Discriminant Analysis Results

\begin{tabular}{llllllll}
\hline HMQ Component & $\begin{array}{l}\text { Wilks' } \\
\text { Lambda }\end{array}$ & Chi-square & df & Significance & \multicolumn{2}{l}{$\begin{array}{l}\text { Percent Correctly Classified by } \\
\text { Discriminant Analysis }\end{array}$} \\
\cline { 3 - 8 } & & & & & Overall & Healthy & Verified Lyme \\
\hline Overall HMQ Score & 0.48 & 522.83 & 1 & $<0.001$ & 87.6 & 90.2 & 81.7 \\
HMQ Sections & 0.34 & 772.57 & 4 & $<0.001$ & 91.2 & 93.8 & 85.4 \\
HMQ Factors & 0.62 & 382.73 & 6 & $<0.001$ & 81.9 & 86.4 & 71.1 \\
\hline
\end{tabular}

Abbreviation: HMQ, Horowitz Multiple Systemic Infectious Disease Syndrome Questionnaire.

since this factor of the HMQ Symptom Checklist contains many items indicative of inflammation and illness that are not limited to Lyme disease.

\section{Predicting patient status - discriminant analysis}

Discriminant analysis was used to test whether HMQ scores could accurately classify individuals into verified Lyme patient vs healthy categories. The following three separate tests were conducted: the overall HMQ Score, scores on the sections of the HMQ, and the HMQ factors. Table 10 shows the results for all three discriminant analyses.

Discriminant analysis for the overall HMQ score showed that it accurately predicted the Lyme category for $87.6 \%$ of the participants. Accuracy for the healthy group was $90.2 \%$ and accuracy for the verified Lyme patient group was $81.7 \%$.

A second discriminant analysis was conducted examining the sections of the HMQ (the HMQ Symptom Checklist, the Lyme Incidence score, the Healthy Days score, and the Common Lyme score). Taken together, the sections of the HMQ accurately predicted the healthy vs Lyme category. The overall accuracy was $91.2 \%$. The HMQ factor scores showed an $83.4 \%$ accuracy rate. These factors were then entered using a stepwise procedure. In the first step, the model selected the factor with the best categorization accuracy and proceeded to enter additional factors in subsequent steps until the remaining factors did not add significantly to the categorization accuracy.
Four of the six factors contributed to the categorization accuracy. The factor entered first was the cognitive dysfunction factor. The musculoskeletal pain factor was entered second, the fatigue factor was entered third, and the dysautonomia factor was entered fourth. The neuropathy and cardio/respiratory factors were not entered into the model. Overall, these four factors led to $82.4 \%$ of the sample being correctly categorized into the verified Lyme patient and healthy groups.

\section{Exploratory analysis on the migratory symptoms items on the Lyme Incidence scale}

As indicated in the results for Study 2, there were three items on the Lyme Incidence scale that focused on migratory symptoms. A chi square analysis showed that migratory symptoms predicted patient status $\left(\chi^{2}[1]=247.15, P<0.001\right)$. As shown in Figure 1, 85.5\% of verified Lyme patients indicated that they experienced at least one of the migratory symptoms, while only $24.7 \%$ of the healthy individuals did.

Similarly, we compared patient status with whether an individual experienced 1, 2, or 3 migratory symptoms. Again, the chi square analysis showed a significant relationship between patient status and migratory symptoms $\left(\chi^{2}\right.$ $[3]=317.72, P<0.001)$. Verified Lyme patients reported more migratory symptoms than healthy individuals. Approximately $51.5 \%$ of verified Lyme patients reported experiencing all three types of migratory pain, while only $5.4 \%$ of healthy 


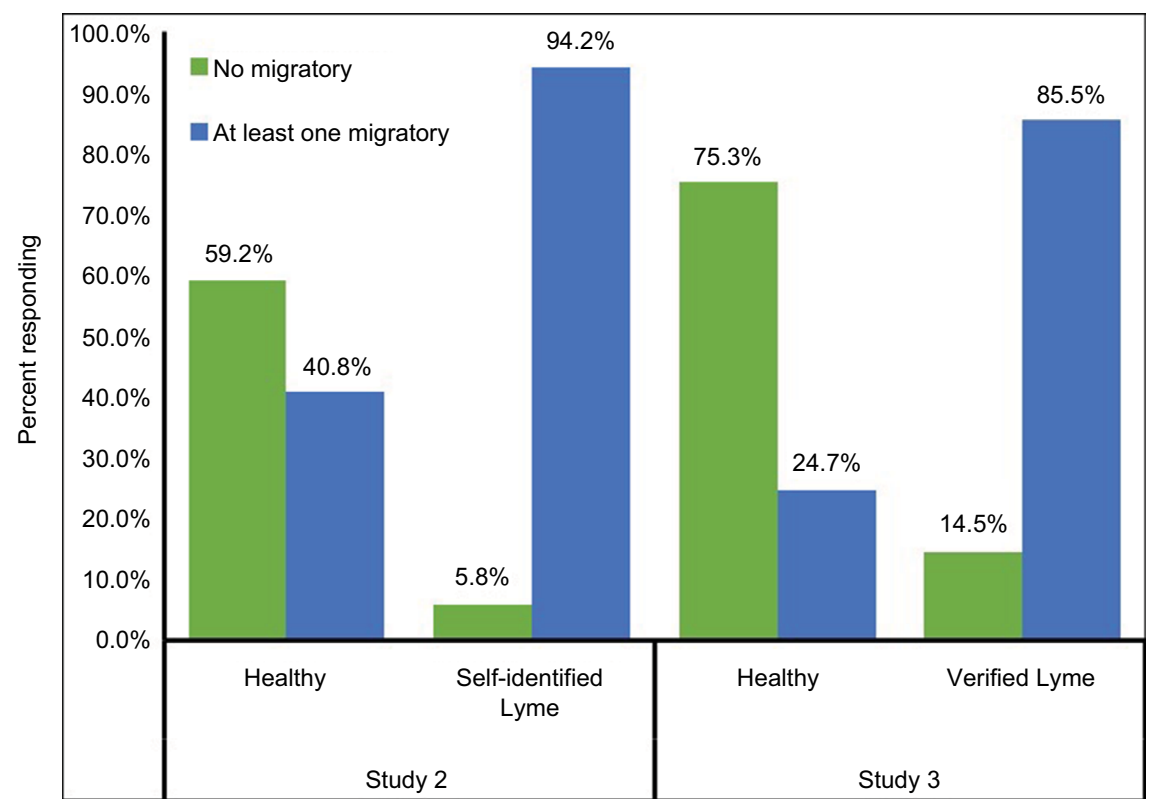

Figure I Migratory symptoms and prediction of healthy vs Lyme status for Studies 2 and 3.

individuals reported experiencing all three types of migratory pain. The majority of healthy individuals $(75.3 \%)$ reported no migratory pain at all.

These results demonstrate the robust ability of the three migratory items on the questionnaire to correctly categorize individuals with Lyme disease.

\section{Discussion}

This article set out to achieve the following four main objectives: 1) to factor analyze the HMQ Symptom Checklist identifying the underlying hallmarks of Lyme disease, 2) to determine the psychometric properties of the HMQ including its reliability and construct validity, 3) to determine whether the HMQ score would show divergent validity, and 4) to determine whether scores on the HMQ would be able to discriminate healthy individuals from those with Lyme disease. The evidence supports these aims and shows that the HMQ demonstrates satisfactory psychometric properties, construct validity, divergent validity, and predictive validity.

The factor analysis identified six underlying latent dimensions, and four of these overlapped with critical symptoms identified by Horowitz in the Common Lyme scale - neuropathy, cognitive dysfunction, musculoskeletal pain, and fatigue. Two additional factors were also revealed - dysautonomia and cardio/respiratory. Of these factors, the cardio/respiratory factor did not appear to contribute as much to identifying those with Lyme from healthy individuals.

The HMQ demonstrated acceptable levels of internal reliability using Cronbach's coefficient alpha with the exception of the Lyme Incidence scale. As discussed previously, this scale appears to combine two different types of questions some regarding possible exposure to ticks that carry the bacteria that causes Lyme disease and those focused on the migratory nature of the symptoms. We recommend that future revisions of this scale move the migratory symptom questions to the HMQ Symptom Checklist and focus the Lyme Incidence scale on potential indicators of exposure to tick-borne diseases.

The results also demonstrated evidence of convergent and divergent validity. HMQ scores correlated more highly with components of the HMQ than with unrelated traits. For example, the Lyme Incidence score correlated more highly with scores on the HMQ Symptom Checklist than with scores from the Big Five personality factors. Correlations between the HMQ scores and the Big Five factors were small and accounted for very little variance. The significant correlations for the neuroticism scale actually fit with recent evidence that suggests negative life events, such as illness, lead to changes in neuroticism scores. ${ }^{56}$ Struggling with the hallmark symptoms of Lyme disease such as cognitive dysfunction, fatigue, and musculoskeletal pain can increase an individual's negative attitude. Higher rates of neuroticism may not reflect an underlying psychiatric problem but merely reflect the chronic stress associated with the illness.

The results demonstrated that the HMQ accurately differentiated Lyme disease patients from healthy individuals. This finding was corroborated with both a sample of selfidentified Lyme sufferers and a sample of verified Lyme 
patients. Means for the overall HMQ score, the HMQ Symptom Checklist, Common Lyme score, and the HMQ factor scores varied significantly by Lyme and patient status. In Study 2, those reporting that they suffered from Lyme now scored higher than healthy individuals. Study 3 showed the same pattern of results with verified Lyme patients scoring significantly higher than healthy individuals. Using discriminant analysis, these scores also produced accurate Lyme status classifications.

Comparing the results from Study 2 and Study 3, the self-identified Lyme group had a higher mean score and a larger standard deviation than the verified Lyme patient sample. This difference might be due to random variation, but it might also be due to the fact that the scale on the HMQ differed between the two studies. The scale in Study 2 focused on severity, and the scale in Study 3 focused on frequency. Across both studies, however, the pattern of findings was similar. Despite whether individuals rated their symptoms in terms of frequency or severity, the HMQ was very accurate in identifying healthy and "Lyme" status. We recommend using the frequency scale used in Study 3. This scale resulted in less inflation in the HMQ scores overall.

\section{Using the questionnaire in clinical practice}

The HMQ may encourage a more holistic view of patient symptoms instead of looking at each complaint in isolation. This broader clinical perspective focusing on a whole constellation of clinical symptoms can provide an early screening tool as well as more accurate patient diagnosis.

Based on the results of the present study, we recommend two uses of the HMQ. First, individuals who suspect that they may have Lyme disease can take the questionnaire. Because of the variety of symptoms, individuals may know that they are not feeling well but may not realize that the combination of these multisystem complaints could be indicative of Lyme. The HMQ empowers them, and the questionnaire provides them with an understanding of how their varied signs and symptoms may be related. This knowledge could be vital in communicating these complaints to a health practitioner.

Second, general practitioners, specialists, and nurses can use the questionnaire to identify individuals with suspected Lyme disease. We propose that for patients who have been diagnosed with diseases without a definitive etiology, such as chronic fatigue syndrome/systemic exertional intolerance disease, fibromyalgia, nonspecific autoimmune disorders (ie, seronegative rheumatoid arthritis), and sudden unexplained neuropsychiatric illness, clinical practitioners use the HMQ score to help rule out Lyme disease or another tick-borne illness.

A key discriminating factor to focus on is the migratory symptoms. These symptoms are a hallmark of Lyme disease but occasionally manifest in other illnesses, ${ }^{65}$ such as inflammatory bowel disorders, gonococcal arthritis, hepatitis, reactive arthritis, acute rheumatic fever, and systemic lupus erythematosus where transient migratory arthritis can be part of the clinical picture. ${ }^{65}$ Table 11 lists the differential diagnosis for migratory pain for each of the above disorders, their clinical characteristics, and suggested laboratory testing. The clinical characteristics of these disorders are significantly different, but when there is a partial overlap of symptoms confusing the clinical picture, appropriate laboratory testing and/or imaging studies listed in Table 11 should help facilitate the diagnostic process. Only Lyme disease presents with persistent migratory pain, especially migratory nerve pain and paresthesias, which are not primary characteristics of these other illnesses.

To identify whether a person is likely to have Lyme disease or not, we recommend the following cutoffs using the overall HMQ score: $<21=$ not likely, $21-36=$ possible, $36-62=$ likely, and $\geq 63=$ highly likely. These cutoffs are based on the mean and standard deviation of scores from Study 3 comparing patients with healthy individuals. The mean score for the healthy group was 20.91, and the standard deviation was 14.75. HMQ scores <21 would be below the mean for this group and suggest that Lyme is not a likely explanation for the individual's symptoms. A score of 36 represents the mean with one standard deviation, while 62 represents the mean of the patient group. Scores falling in this range suggest that follow-up Lyme testing is advisable. Scores $\geq 63$ suggest that the presence of Lyme is strongly indicated and testing is highly recommended.

Scores in the likely or highly likely categories should lead providers to do a broad panel of tick-borne testing. ${ }^{66}$ Testing should include not only an ELISA, which has limited sensitivity, ${ }^{28}$ but also a C6 ELISA ${ }^{67}$ and IgM/IgG Western blots, focusing on the presence of Borrelia-specific bands ${ }^{68}$ to capture the varied species of Borrelia. B. sensu lato $^{69}$ and relapsing fever Borrelia ${ }^{70}$ are now known to also cause chronic illness.

Considering that ticks can transmit a variety of illnesses, the HMQ can highlight symptoms that reflect coinfections. Particularly, high scores and/or key patterns of responses warrant further testing. We propose including a complete blood count $(\mathrm{CBC})$ and comprehensive metabolic profile (CMP) to look for the presence of leukopenia, thrombocytopenia, and 
Table I I MSIDS differential diagnosis of migratory pain

\begin{tabular}{l} 
Clinical characteristics \\
\hline Crohn's disease/IBD ${ }^{\mathbf{a}, 96-98}$ \\
Abdominal pain and tenderness (often right lower quadrant), chronic diarrhea, \\
usually with rectal bleeding, fever, loss of appetite, weight loss with malabsorption \\
Extraintestinal manifestations can include: \\
Dermatologic: EN, PG, apthous stomatitis, fistulas \\
Ocular*: conjunctivitis, uveitis/iritis, episcleritis with ocular pain, photophobia, blurry \\
vision \\
Hepatobiliary: hepatomegaly*, hepatic steatosis/NASH, gallstones, pancreatitis, PSC \\
Cardiopulmonary: endocarditis*, myocarditis*, pericarditis*, vasculitis*, thrombosis \\
(hypercoagulability), interstitial lung disease \\
Renal: amyloidosis, calculi, ureteral obstruction with renal failure \\
Rheumatologic: arthritis develops in I5-20\% of IBD and worsens with active bowel \\
inflammation. Usually involves large joints, and is asymmetric. Ankylosing spondylitis, \\
sacroiliitis, hypertrophic osteoarthropathy, and relapsing polychondritis can be \\
associated \\
Gonococcal arthritis ${ }^{65,99-101}$
\end{tabular}

Bacteremic DGI can have presenting complaints of migratory arthralgias, but symptoms are usually accompanied by papules evolving into hemorrhagic pustules, and/or hemorrhagic bullae near joints ( $75 \%$ of the time). The triad of suppurative arthritis, tenosynovitis and dermatitis helps identify DGI. Polyarthralgias usually involve knees, wrists, elbows, ankles, and distal joints. Rarely osteomyelitis is present

Extrarheumatic manifestations include:

Genitourinary: mucopurulent cervicitis (women), urethritis with urethral discharge and dysuria (men); rare: epididymitis, prostatitis, vesiculitis, balanitis, fistulas Gastrointestinal: purulent rectal discharge, proctitis with pain, bleeding

Dermatologic: nodules, EN, urticaria

Pharyngitis* with cervical lymphadenitis*

Ocular: conjunctivitis*, purulent discharge, corneal ulcerations/ophthalmia neonatorum (complications in neonates during parturition)

Cardiac: endocarditis* (rare)

Neurologic: meningitis* (rare)

Hepatitis (A, B, C, D, E) (5, $102-106^{-10}$

All types of hepatitis viruses cause clinically similar illnesses. Symptoms range from being asymptomatic, to fulminant acute infections with hepatic necrosis.

Blood-borne types (HBV, HCV, HDV) can cause subclinical persistent infections or rapidly progress to chronic liver disease with cirrhosis and hepatocellular carcinoma Liver manifestations: hepatomegaly with right upper quadrant pain, occasional splenomegaly; abnormalities in liver function testing, with jaundice (Tickborne co-infections, such as relapsing fever borrelia, ehrlichiosis, anaplasmosis, rickettsial infections, tick-borne viruses and babesiosis can also cause similar liver abnormalities)

Cirrhosis can present with ascites, peripheral edema, esophageal bleeding and hepatic encephalopathy. Palmar erythema, gynecomastia and spider angiomas suggest chronic liver disease

Extrahepatic manifestations (associated with circulating immune complexes) can lead to:

Prodromal serum sickness: anorexia, nausea, vomiting (rarely associated with pancreatitis), mild weight loss, fatigue, fever, arthralgias, myalgias, headache, photophobia, cough, coryza, pharyngitis with alterations in taste and olfaction Kidney: glomerulonephritis, nephrotic syndrome, dark urine (and clay colored stools) before onset of jaundice

Cardiovascular: vasculitis (polyarteritis nodosa), palpable purpura, rarely

myocarditis*, pneumonia

Other neuro: peripheral neuropathy* (rare)

Arthritis: can be associated with EMC but is not a prominent feature of the overall clinical picture

Laboratory/clinical evaluation

Stool samples for blood and/or infectious microbes

$\mathrm{CBC}, \mathrm{CMP}$ with liver functions, homocysteine, coagulation

profile, HLA-B27, pANCA

Imaging studies:

CT scans, barium swallow, video capsule endoscopy, colonoscopy

Abdominal ultrasound ERCP or MRCP if jaundice (PSC)

Culture (urogenital specimens, synovial fluid), urethral swabs with gram stain, NAATs, DNA probes

Blood cultures/screen for complement deficiency with total hemolytic complement activity if DGI

IgM (acute), IgG (chronic) antibodies, Ags:

$\mathrm{HAV}$ (anti-HaV)

Anti-HBs, HBsAg, HBV DNA

Anti-HBc, $\mathrm{HBcAg}$

Anti-HBe, $\mathrm{HBeAg}$

Anti-HCV, HCV RNA

Anti-HDV, HDV Ag

Anti-HEV, HEV Ag

Liver function testing: elevations in AST, ALT, serum bilirubin. Serum alkaline phosphatase may be normal or slightly elevated $\mathrm{CBC}$ may show a transient neutropenia, and lymphopenia, followed by lymphocytosis with atypical lymphocytes.

PT: may be prolonged and follow alpha-I-antitrypsin levels (hepatocellular carcinoma)

Autoantibodies (ANA, RF, smooth muscle) are possible (acute)

Urine: microscopic hematuria, slight proteinuria (unless nephrotic syndrome) 
Table I I (Continued)

Clinical characteristics $^{\text {a }}$

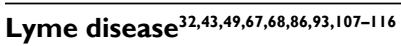

Multisystemic symptoms often tend to come and go, with good and bad days, intensified by hormonal changes (menses) and/or antibiotic use (improvement in symptoms or worsening secondary to Herxheimer reactions)

Rashes: EM rashes are present approximately $50 \%$ of the time (USA), with higher incidences reported in Europe. Not all rashes resemble a "bull's eye" pattern; a solid spreading rash is also possible; other Borrelia species (Borrelia mayonii) can cause a wide-spread spotted rash. Borrelia afzelii causes ACA, a violaceous skin rash of the extremities, more often seen in Europe. Morgellon's disease or "fiber" disease with open wounds and multisystemic symptoms has also been associated with Borrelia infection

General: fatigue, flu-like symptoms, pharyngitis with cervical lymphadenopathy Rheumatologic: migratory joint and muscle pain are commonly seen in both early and late Lyme disease. The migratory pain can be either symmetric or asymmetric, affecting large and small joints. Direct signs of inflammation (heat, tenderness to palpation), can be present, as can swelling of the joints (synovitis with synovial effusions), but these signs are not necessarily seen in all patients with multisystemic complaints. The pain may migrate over hours or days, in both acute and late stage disease. Nonmigratory pain can also be present, as in the case of acute monoarticular arthritis (oftentimes affecting the knee post tick-bite in children $\leq 18$ years of age).

Cardiopulmonary: chest pain (usually caused by costochondritis), palpitations, and shortness of breath are commonly reported (rule out babesiosis, relapsing fever, cardiomyopathy). Carditis with third degree heart block, myocarditis, endocarditis and pericarditis are rare complications.

Neurologic: nerve pain (neuropathy) which frequently has a migratory component is a common manifestation of both PNS and CNS borreliosis. Symptoms are usually described as burning, tingling, stabbing, crawling sensations and/or numbness (occasionally associated with small fiber neuropathy). The quality of the pain often changes with antibiotic treatment (improvement, or worsening with a Herxheimer reaction). Mononeuropathies (mononeuropathy multiplex), polyneuropathies, radiculitis, radiculoneuritis with pain, loss of motor control, and sensory deficits are often associated with PNS involvement. CNS involvement may cause a lymphocytic meningitis, encephalitis, myelitis and vasculitis.

Common neurologic symptoms include a stiff neck, headache, light and sound sensitivity, dizziness, cognitive difficulties (memory and concentration problems, word finding problems with difficulty in executive functioning), and sleep disruption (DSPS with difficulty falling asleep and staying asleep).

Cranial nerve palsies oftentimes involve the 2 nd (optic neuritis), 5th (trigeminal) and 7th cranial nerve (Bell's palsy), but all cranial nerves can be affected. Bannwarth syndrome is a triad of radiculitis, Bell's palsy and meningitis, associated with neuroborreliosis (Borrelia garinii [Europe] and Borrelia burgdorferi [USA]).

Neuropathy of the ANS can cause dysautonomia/POTS, affecting the blood pressure (postural orthostasis with dizziness standing), heart rate (tachycardia), sweat glands (anhidrosis, hyperhidrosis), and bladder and bowel dysfunction (difficulty urinating, gastroparesis, chronic constipation). Antiganglioside antibodies may be present. Rare neurological complications may include an ALS type picture with a polyradiculopathy.

Psychiatric: a broad range of psychological manifestations (depression, anxiety,

OCD, rarely psychosis) have been reported.

Ophthalmologic: conjunctivitis, uveitis, retinitis, or optic neuritis

Genitourinary/GYN: detrusor dysfunction, interstitial cystitis, vaginal neuralgia, dyspareunia

Gastrointestinal: nausea, vomiting, abdominal pain, anorexia, rare diarrhea (early Lyme disease)
Laboratory/clinical evaluation

Use a panel approach with indirect and direct tests to confirm a clinical diagnosis:

Indirect tests:

- ELISA

- C6 ELISA (preferred in European patients and US patients, as it includes $B$. afzelii, $B$. garinii, and $B$. burgdorferi).

- IFA

- IgM and IgG Western blots (pay attention to Borrelia specific bands: 23 [OspC], 31 [OspA], 34 [OspB], 39, 83/93). These can be present with different Borrelia species.

- ELISpot, Lymphocyte Transformation Test (LTT)

- Spirotest (CXCL9, CXCLI0, CCLI9)

Direct tests:

Nanotrap (urine Ag testing with OspA)

PCR

Culture

Other testing: other Borrelia species (including relapsing fever, B. miyamotoi) can cause Lyme-like syndromes. Check antibodies, PCR

Check coinfection testing if there is a malaria-like illness (Babesia spp.), atypical skin rashes resembling "stretch marks" (Bartonella spp.), and/or severe atypical clinical manifestations (rule out rickettsia, Q-fever, tularemia)

Tick-borne viruses should be checked if severe neurological manifestations are present (TBE virus [Europe], Deer tick virus, and Powassan [USA])

Autoantibodies are frequently present in Lyme borreliosis, including positive ANAs, and RFs which are surrogate markers of inflammation. These titers oftentimes decrease or resolve with appropriate antibiotic treatment.

Check an anti-dsDNA and anti-Sm (positive in SLE) and antiCCP (anticyclic citrullinated peptide, positive in rheumatoid arthritis) to rule out true SLE and rheumatoid arthritis in a patient with a multisystemic disorder and migratory pain with autoantibody production.

Patients with autoimmune diseases may also be exposed to Lyme disease and tick-borne co-infections, so it is necessary to rule out overlapping pathologies.

Check antiganglioside antibodies if neuropathic syndromes/ dysautonomia is present. 
Table I I (Continued)

Clinical characteristics $^{\mathbf{a}}$
Hepatic: hepatosplenomegaly, transaminitis (early disease; rarely in late disease).
Differential diagnosis for Gl symptoms and transaminitis includes overlapping tick-
borne diseases, including: anaplasmosis, ehrlichiosis, relapsing fever bacteria (Borrelia
hermsii, Borrelia miyamotoi), rickettsial infections (Rocky Mountain spotted fever,
Q fever [Coxiella burnetii], Tick-borne viruses (Heartland, Bourbon viruses), and
babesiosis.
Other Borrelia species like B. hermsii can cause nausea, vomiting, abdominal pain,
hepatitis, jaundice, and an enlarged spleen
ReA

Acute, nonpurulent arthritis usually following an enteric infection (Salmonella, Shigella, Yersinia, Camplylobacter spp., as well as Clostridium difficile) or urogenital infection (Chlamydia trachomatis and other species including Ureaplasma urealyticum and Mycoplasma genitalium). Occasionally respiratory infection with Chlamydia pneumoniae is implicated

Often associated with HLA-B27, which significantly prolongs the survival of intracellular bacteria.

Symptoms often occur I-4 weeks after the infection.

Triad of arthritis, urethritis, and conjunctivitis is one part of the clinical spectrum, which ranges from a transient monoarthritis to severe multisystem disease with fatigue, fever and weight loss.

Arthritis is usually painful, asymmetric, and additive. New joint involvement can occur over several days to weeks and typically lasts 3-5 months (rarely up to one year). Dactylitis (sausage digit), with diffuse swelling of a finger or toe is a classic manifestation, with tense joint effusions. Tendonitis and fasciitis often produce pain at insertion sites (entheses). Low back pain is also often seen, with or without a sacroiliitis Urogential involvement: urethritis, prostatitis, cervicitis, salpingitis

Ocular: transient conjunctivitis, anterior uveitis

Dermatologic: mucocutaneous lesions are common, including oral ulcers; characteristic skin lesion is keratoderma blenorrhagica, with vesicles on the palms and soles (most common sites). Nail changes are common (hyperkeratosis, yellowish discoloration, onycholysis).

Cardiopulmonary: aortic insufficiency, conduction defect, pleuropulmonary

infiltrates (rare)

Neurologic: CNS and/or PNS lesions (rare)

ARF $65,118-120$

Symptoms appear I-5 weeks after a group A strep infection. Involves major and minor manifestations.

Major manifestations: polyarthritis*, carditis*, chorea, erythema marginatum, subcutaneous nodules.

Minor manifestations: fever* and polyarthralgia*, inflammatory markers (elevated sedimentation rate, white cell count), and a prolonged PR interval on the electrocardiogram

Most common presentation is fever and an inflammatory polyarthritis. Large joints are typically affected (hips, knees, ankles, elbows) in a migratory, asymmetric fashion (60-75\% cases). Migratory pain is over a period of several hours

Cardiac: carditis is the next most common clinical feature, seen in $50-60 \%$ of cases. Chronic valvular lesions of rheumatic heart disease usually involve the mitral valve (mitral stenosis) or mixed mitral valve disease and/or aortic valve disease Neurologic: Sydenham's chorea ( $<2-30 \%$ cases), can occur without other clinical signs and symptoms. Usually resolves within 6 weeks.

Dermatologic: erythema marginatum and subcutaneous nodules are rare $(<5 \%$ cases). Erythema marginatum is the classic rash of ARF. It consists of pink macules which clear in the center, leaving a serpiginous edge. They can be differentiated from the classic rash of Lyme disease (EM) as they quickly appear and disappear. *Polyarthritis and carditis are seen in Lyme disease, but the arthritic pain in Lyme disease affects large and small joints and can be symmetric or asymmetric, migrating over hours and days. Lyme carditis is a rare manifestation.

Laboratory/clinical evaluation

HLA-B27

Serological testing for triggering infections: check antibodies against Yersinia, Salmonella, Chlamydia, etc., with culture (Yersinia, Chlamydia), and PCR Check inflammatory markers (ESR, CRP), and a CBC for anemia

X-ray: periostitis with reactive new bone formation is characteristic of ankylosing spondylitis

Blood tests and $\mathrm{X}$-rays only help to confirm a clinical diagnosis in an individual with an acute inflammatory, asymmetric, additive arthritis or tendinitis, with extraarticular involvement (eyes, mucous membranes, skin, nails, genitalia)

Diagnosis is made based on the constellation of clinical symptoms after a group $A$ strep infection.

Throat swab culture or rapid Ag testing may be negative, so serologic testing with an ASO and ADB titers should also be performed

Jones criteria are used to aid in the diagnosis. Two major or one major and two minor manifestations plus evidence of a preceding group A streptococcal infection are necessary to establish the diagnosis for the primary episode of ARF. Testing should also include a white blood cell count, ESR, CRP, and blood cultures if febrile

Electrocardiogram, echocardiogram, and chest $\mathrm{X}$-ray if clinical suspicion of carditis

Differential diagnosis includes PSRA, which affects small joints, often symmetrically, without carditis, and PANDAS, causing tics and obsessive-compulsive symptoms. 
Table I I (Continued)

\begin{tabular}{|c|c|}
\hline Clinical characteristics $^{a}$ & Laboratory/clinical evaluation \\
\hline \multicolumn{2}{|l|}{ SLE $^{65,121-124}$} \\
\hline Diagnosis is made based on both clinical symptoms and laboratory evidence of & High titers of double stranded DNA (Anti-dsDNA) and Sm \\
\hline autoantibodies. & $\mathrm{Ag}$ (anti-Sm) are highly specific for SLE and should be used to \\
\hline A combination of $>4$ of the II criteria listed below increases the likelihood of SLE & confirm a clinical diagnosis \\
\hline ( $\sim 95 \%$ specificity, $\sim 75 \%$ sensitivity): & Other autoantibodies are oftentimes present, but are not \\
\hline Malar rash (butterfly appearance)* & specific for SLE: \\
\hline Discoid rash (circular raised patches with keratosis and/or scarring) & ANA* \\
\hline Photosensitivity: exposure to ultraviolet light causes a rash & Anti-RNP* \\
\hline Nasopharyngeal ulcers & Antihistone antibodies \\
\hline Arthritis: in $\geq 2$ peripheral joints, which is nonerosive. Tenderness and swelling with & Antiphospholipid antibodies* \\
\hline or without an effusion is required* & Anti-Ro (anti-SS-A)* \\
\hline Serositis: pleuritis (with or without pleural effusion), or pericarditis (documented by & Anti-La (anti-SS-B)* \\
\hline a rub on auscultation, or an electrocardiogram or echocardiogram)* & Antierythrocyte antibodies (direct Coombs test) \\
\hline Hematological abnormalities: anemia (hemolytic), leukopenia $(<4000 / \mathrm{mL})$ or & Antineuronal antibodies (antiglutamate receptor)* \\
\hline lymphopenia $(<1500 / \mathrm{mL})$ or thrombocytopenia $(<100,000 / \mathrm{mL})^{*}$ & Antiribosomal P \\
\hline Renal: proteinuria $(>0.5 \mathrm{~g} /$ day $)$ or casts & Initial laboratory evaluation: \\
\hline Neurological: seizures, psychosis (without other causes)* & CBC, platelets \\
\hline Immunological disorder: anti-dsDNA, anti-Sm; and/or antiphospholipid antibodies* & CMP with renal function \\
\hline ANAs (not drug induced)* & Urinalysis \\
\hline Symptoms can be mild or severe, with intermittent exacerbations. & ANA, ds-DNA, anti-Sm \\
\hline The most common symptoms of SLE include: & Anti-Ro \\
\hline Fatigue* and arthralgias/myalgias* (usually present most of the time) & Use ds-DNA and anti-Sm to help differentiate the two \\
\hline Fever* & diseases since both SLE and Lyme disease can result in positi \\
\hline Headaches* & ANAs, antiphospholipid antibodies and other autoimmune \\
\hline Hair loss* & markers \\
\hline Malar shaped rash* & Migratory symptoms (arthritis, myalgias, and/or neuropathy) \\
\hline Photosensitivity & associated with a broad range of abnormalities of the PNS \\
\hline Intermittent polyarthritis (mild to severe) usually in hands, wrists, and knees* & and CNS (refer to neurological Lyme disease symptoms) are \\
\hline Pleurisy & more commonly seen in Lyme disease than in SLE. Migratory \\
\hline Anemia & neuropathy (tingling, numbness, burning, stabbing sensations) \\
\hline Edema (extremities, periorbital) renal disease and nephrotic syndrome & is a prominent symptom of Lyme disease, not usually present \\
\hline Abnormal blood clotting (antiphospholipid syndrome) & in other illnesses. \\
\hline
\end{tabular}

Mouth or nose ulcers

Raynaud's phenomenon

(Starred symptoms and/or laboratory testing [*] are nonspecific and can be seen with Lyme disease, which is often associated with autoantibody production). Oral ulcers, pleurisy, malar rashes, abnormal blood clotting, renal disease, and edema are not usually associated with Lyme disease.

Overlapping syndromes: seizures and psychosis can be seen with Lyme disease, but are rare clinical manifestations.

Anemia and photosensitivity if present in Lyme disease are usually due to medication side effects.

Tick-borne coinfections such B. miyamotoi, ehrlichiosis, anaplasmosis, rickettsial infections, and babesiosis can cause leukopenia and thrombocytopenia and should be included in the differential diagnosis if hematological abnormalities are present.

Notes: a These clinical characteristics represent common disease manifestations, not a full comprehensive list.

Abbreviations: ACA, acrodermatitis chronicum atrophicans; ADB, anti-DNase B; Ag, antigens; ALS, amyotrophic lateral sclerosis; ALT, alanine transaminase; ANAs, antinuclear antibodies; ANS, autonomic nervous system; ARF, acute rheumatic fever; ASO, antistreptolysin O; AST, aspartate transaminase; CBC, complete blood count; CMP, comprehensive metabolic profile; CNS, central nervous system; CRP, C-reactive protein; DGI, disseminated gonococcal infection; DSPS, delayed sleep phase syndrome; $E M$, erythema migrans; EMC, essential mixed cryoglobulinemia; EN, erythema nodosum; ERCP, endoscopic retrograde cholangiopancreatography; ESR, erythrocyte sedimentation rate; GI, gastrointestinal; GYN, gynecologic; HAV, hepatitis A; HBV, hepatitis B; HCV, hepatitis C; HDV, hepatitis D; HEV, hepatitis E; HLA-B27, human leukocyte antigen-B27; IBD, inflammatory bowel disease; IFA, immunofluorescent antibodies; MRCP, magnetic resonance cholangiopancreatography; MSIDS, Multiple Systemic Infectious Disease Syndrome; NAATs, nucleic acid amplification tests; NASH, nonalcoholic steatohepatitis; OCD, Obsessive-Compulsive Disorder; pANCA, perinuclear antineutrophil cytoplasmic antibodies; PANDAS, pediatric autoimmune neuropsychiatric disorders associated with streptococcal infection; PCR, polymerase chain reaction; PG, pyoderma gangrenosum; PNS, peripheral nervous system; POTS, postural orthostatic tachycardia syndrome; PSC, primary sclerosing cholangitis; PSRA, poststreptococcal reactive arthritis; PT, prothrombin time; ReA, reactive arthritis; RFs, rheumatoid factors; RNP, ribonucleoprotein; SLE, systemic lupus erythematosus; SS-A, Sjögren's syndrome-related antigen A; SS-B, Sjögren's syndrome-related antigen B; TBE, tick-borne encephalitis. 
transaminitis, as these can occur with Ehrlichia, Anaplasma, rickettsial infections, ${ }^{71}$ as well as Borrelia species including B. miyamotoi. ${ }^{72}$ Other tick-borne infections like babesiosis should also be considered in severely ill patients, ${ }^{73}$ since it is frequently transmitted at the same time as borreliosis. ${ }^{74}$ Any patient who complains of unexplained fevers, day and night sweats, chills, flushing, an unexplained cough, and shortness of breath (air hunger), which are questions number 1 and number 22 (Section 1 of the HMQ), may also have a concomitant infection with babesiosis. ${ }^{75-77} \mathrm{~A}$ Babesia panel approach with a Giemsa stain, Babesia titers (IFA) for multiple species of Babesia (Babesia microti, Babesia duncani [WA-1, Babesia divergens and Babesia sp. EU1]), with PCR [61] and FISH testing, may help to establish the diagnosis ${ }^{78}$ in the United States and Europe, ${ }^{78}$ while ruling out other causes of these symptoms. Other potential tick-borne transmitted infections, including Bartonella ${ }^{79}$ and tularemia ${ }^{80}$ should also be considered in the differential diagnosis in those experiencing an unexplained chronic fatiguing, musculoskeletal illness, as well as brucellosis, which has been found in a small percentage of individuals from the Midwest USA diagnosed with CFS. ${ }^{81}$

\section{Limitations and issues to be considered}

Symptoms in early and late Lyme are similar, ${ }^{19}$ although longterm infection "can result in advanced disease involving the heart, nervous system, or joints." ${ }^{19}$ Recent scientific evidence has demonstrated that Borrelia is able to persist after standard courses of antibiotics. ${ }^{82-85}$ One potential way to use the HMQ early and later in the disease process where antibody testing may be negative is to identify individuals with a high HMQ score and multisystemic illness and evaluate them with a broad tick-borne testing panel (C6 ELISA, IFA, IgM and IgG Western blots, PCR, Lymphocyte Transformation Test, Lyme Dot Blot, and Nanotrap test), ${ }^{86}$ including using recently identified inflammatory chemokines characteristic of those with an acute and later stage Borrelia infection. ${ }^{32,87}$

The larger question remains: How do you reliably identify Lyme sufferers from healthy individuals?; what is healthy?; is it someone who has never been exposed to the Bb bacteria?; and is it someone who has been exposed, but has received appropriate treatment and now is symptom free? The results of the present study demonstrated that the HMQ score can discriminate between individuals with documented Lyme (through blood tests and EM rashes) and healthy individuals. Additional research is needed to determine whether high HMQ scores can distinguish between those with Lyme disease and those who are sick from other illnesses.
Migratory symptoms on the HMQ helped identify those verified to have Lyme disease. Symptoms that are migratory and come and go are classic manifestations of early disseminated Lyme disease, ${ }^{19}$ as identified by Steere in $2001 .^{88}$ We know of no other validated screening tool that identifies migratory symptoms as potential hallmarks of both early and late Lyme disease. This is important, since Lyme can also cause autoimmune manifestations producing positive antinuclear antibodies (ANAs) and rheumatoid factors with joint and muscle pain, leading a clinician to believe that the patient suffers from lupus or rheumatoid arthritis. Additional research specifically examining the use of migratory symptoms to predict Lyme infection is warranted.

The aim of Study 3 was to examine whether the HMQ can distinguish between healthy individuals and those with Lyme. Results showed strong support for this aim. We did not examine whether scores on the HMQ could distinguish between Lyme disease and other diseases such as fibromyalgia, chronic fatigue, Alzheimer's disease, and rheumatoid arthritis, and future researchers may want to examine this question. Also, we do not yet know how the gestalt of symptoms identified on the HMQ is affected by overlapping medical problems, which can be exacerbated by Lyme and tick-borne diseases. ${ }^{43,86}$ Our results demonstrate the HMQ's clinical value as an initial prescreening tool. We conclude that the HMQ can assist patients and providers by highlighting those symptoms that require further evaluation. A prescreening tool that is inexpensive and easy to administer with demonstrated validity can improve detection and health outcomes.

Results of Study 2 are particularly important for this purpose. It shows that there are variations in HMQ scores and patterns of responding corresponding to the recognition that a person may have Lyme and these match the pattern of responses of verified Lyme patients from Study 3. Study 2 participants represent the target population where a prescreening tool would have its greatest utility.

Researchers may also want to examine whether patients receiving treatment report lower HMQ scores than those seeking treatment for the first time. A longitudinal study that follows patients through the course of their treatment comparing HMQ scores over time would be an important follow-up. A study of this type could also examine how well individuals respond to treatment and can compare early detection vs late detection. Can the HMQ detect cases where initial treatment did not resolve the underlying disease and further follow-up is necessary? 
Implications of these results also raise additional research questions. Would scores on the HMQ reflect persistent symptoms that respond less well to treatment? Do these persistent symptoms reflect coinfections? Furthermore, can the HMQ be used as an effective screening tool for high risk populations living in endemic areas? This could include patients in multiple specialty practices, including OB-GYN, cardiology, neurology, and psychiatry, where some of the symptoms on the questionnaire would not be part of the routine clinical anamnesis.

\section{Recommendations for revision of the scale}

Based on the results presented here, we suggest several revisions that could improve the HMQ. First, we recommend that the number of items be reduced based on the factor analysis. This would create an easily administered, shortened version for prescreening. A shortened version would be beneficial because of the predominance of cognitive dysfunction among individuals with Lyme disease whose cognitive impairments make focusing, attention, and reading particularly difficult. The concentration required to respond to the 38-symptom checklist may not be practical. If practitioners felt it necessary, they could follow up with the longer version as a diagnostic tool.

One suggestion for pruning the scale may be to remove items with high levels of not applicable responses. These items may be useful for practitioners' doing general health screenings but may not be particularly useful in a prescreening tool for Lyme disease. Consideration should be given as to whether these items should be dropped or remain on the scale. For a short screening tool, our recommendation is to drop them. For a long-form version of the scale, our recommendation is to keep them but remove them from the calculation of the HMQ score.

Another suggestion is to merge the three migratory symptom items (ie, migratory joint pain, migratory muscle pain, and migratory tingling and burning) from the Lyme Incidence section with their corresponding items on the HMQ Symptom Checklist. These items were reported more often by self-identified Lyme sufferers and verified Lyme patients. The migratory nature of these symptoms is both a classic hallmark of Lyme disease and a factor that helps distinguish between Lyme disease and other illnesses. Moving these items would also likely increase the reliability of the Lyme Incidence scale. We also recommend separating out the two questions, as to whether there was a tick-bite with a rash, or a tick-bite followed by flu-like symptoms. Any of these changes to the HMQ would require validation of a revised version and new recommended score cut-offs.

\section{Conclusion}

Lyme and associated tick-borne illness is currently spreading, with constant rates of geographic expansion in all accessible directions in the United States. ${ }^{6}$ Tools that can improve the early detection of Lyme can assist in effective diagnosis and treatment. Lyme disease in its early stage can be effectively treated, but persistent infection, complex pathology affecting multiple body systems and organs, biofilm and cyst formation, and associated tick-borne coinfections make chronic Lyme/PTLDS difficult to diagnose and complex to treat. ${ }^{89-93}$

Lyme disease remains a clinical diagnosis as per the CDC. ${ }^{94}$ As previous research has demonstrated, self-reported symptoms can be reliable predictors of health outcomes. ${ }^{95}$ Assessing clinical symptoms is a hallmark of medical diagnostic strategy. There is also a long history of using validated self-report questionnaires for detecting/diagnosing medical and mental health conditions and quality of life issues in medicine. Validated questionnaires are used extensively to diagnose clinical depression, anxiety disorders, GERD, dysphagia, peripheral artery disease, etc.

The HMQ's utility lies in its ability to serve as a prescreening device to aid individuals in recognizing the symptoms of Lyme and the need to seek treatment from their health care practitioner. It is efficient and inexpensive. Providers can administer the HMQ quickly during an individual's annual checkup and during follow-up examinations using the questionnaire to identify symptoms that are part of a multisystemic disease profile. We recommend using the validated HMQ as part of routine health screening practices facilitating medical judgments about whether a person should undergo further testing to determine exposure to $\mathrm{Bb}$ or other tick-borne illnesses.

\section{Acknowledgments}

We acknowledge and thank Sonja Siderias, LPN, Renee Nelson, Haley Moss Dillon, PhD, and our health care colleagues at Sojourns Community Health Clinic, Westminster, VT, USA, and Naturopathic Health Center, Southbury, CT, USA, for their assistance with our research. We would also like to thank Christina Covington for her help conducting a pre-test study and the SUNY New Paltz Summer Undergraduate Research Experience (SURE) for providing funding for that summer program. The authors thank Basant Puri and Sunny Duerr for their valuable statistics suggestions and the three anonymous reviewers of this article for their very helpful comments and recommendations. 


\section{Disclosure}

Dr Phyllis R Freeman is a senior researcher and Dr Richard Horowitz is an owner and medical practitioner at the Hudson Valley Healing Arts Center, which specializes in the treatment of Lyme disease. Dr Richard Horowitz is also an author of the 2013 book, Why Can't I Get Better? Solving the Mystery of Lyme and Chronic Disease and the 2017 book How Can I Get Better? An Action Plan for Treating Resistant Lyme \& Chronic Disease from St. Martin's Press. The authors report no other conflicts of interest in this work.

\section{References}

1. Smith AJ, Oertle J, Prato D. Chronic Lyme disease: persistent clinical symptoms related to immune evasion, antibiotic resistance and various defense mechanisms of "Borrelia burgdorferi.". Open J Med Microbiol. 2014;4(4):252-260.

2. Berndtson $\mathrm{K}$. Review of evidence for immune evasion and persistent infection in Lyme disease. Int J Gen Med. 2013;6:291-306.

3. Hofhuis A, Harms M, Bennema S, van den Wijngaard CC, van Pelt W. Physician reported incidence of early and late Lyme borreliosis. Parasit Vectors. 2015;8(1):161.

4. Hofhuis A, Harms M, van den Wijngaard C, Sprong H, van Pelt W. Continuing increase of tick bites and Lyme disease between 1994 and 2009. Ticks Tick Borne Dis. 2015;6(1):69-74.

5. Newman EA, Eisen L, Eisen RJ, et al. Borrelia burgdorferi sensu lato spirochetes in wild birds in Northwestern California: associations with ecological factors, bird behavior and tick infestation. PLoS One. 2015;10(2): 0118146.

6. Kugeler KJ, Farley GM, Forrester JD, Mead PS. Geographic distribution and expansion of human Lyme disease, United States. Emerg Infect Dis. 2015;21(8):1455-1457.

7. ECDC. Lyme borreliosis in Europe; 2017. Available from: http://ecdc. europa.eu/en/healthtopics/vectors/world-health-day-2014/Documents/ factsheet-lyme-borreliosis.pdf. Accessed July 7, 2017.

8. Sykes RA. An Estimate of Lyme borreliosis incidence in Western Europe. Res Medica. 2014;22(1):76.

9. Centers for Disease Control and Prevention [webpage on the Internet]. How Many People Get Lyme disease? / Lyme Disease. CDC. Available from: http://www.cdc.gov/lyme/stats/humancases.html. Accessed August 19, 2016.

10. Hook S, Nelson C, Mead P. Self-reported Lyme disease diagnosis, treatment, and recovery: Results from 2009, 2011, \& 2012 HealthStyles nationwide surveys. Presented at the 13th International Conference on Lyme Borreliosis and other Tick-Borne Diseases, Boston, MA, August 19, 2013.

11. Adrion ER, Aucott J, Lemke KW, Weiner JP. Health care costs, utilization and patterns of care following Lyme disease. PLoS One. 2015;10(2):e0116767.

12. Zhang X, Meltzer MI, Peña CA, Hopkins AB, Wroth L, Fix AD. Economic impact of Lyme disease. Emerg Infect Dis. 2006;12(4):653-660.

13. Johnson L. Insurance and Lyme Disease: A Problem of Displaced Costs; 2013. Available from: https://www.lymedisease.org/wp-content/ uploads/2015/02/Chart-book-11.14.13-FINAL-with-correction.pdf. Accessed August 24, 2016.

14. Ahern H. Comparison of Lyme disease prevalence and disease reporting in an endemic area. J Microbiol Res. 2013;3(6):261-265.

15. van den Wijngaard CC, Hofhuis A, Wong A, et al. The cost of Lyme borreliosis. Eur J Public Health. 2017;27(3):538-547.

16. van den Wijngaard CC, Hofhuis A, Harms MG, et al. The burden of Lyme borreliosis expressed in disability-adjusted life years. Eur $J$ Public Health. 2015;25(6):1071-1078.
17. Fallon BA, Levin ES, Schweitzer PJ, Hardesty D. Inflammation and central nervous system Lyme disease. Neurobiol Dis. 2010;37(3):534-541.

18. White J, Noonan-Toly C, Lukacik G, et al. Lyme disease surveillance in New York State: an assessment of case underreporting. Zoonoses Public Health. Epub 2016 Sep 10.

19. DePietropaolo DL, Powers JH, Gill JM, Foy AJ. Diagnosis of Lyme disease [published correction appears in Am Fam Physician, 2006; 73(5):776]. Am Fam Physician. 2005;72(2):297-304.

20. Meyerhoff J, Zaidman G, Steele R, Diamond H [webpage on the Internet]. Lyme Disease Workup: Approach Considerations, Serologic Testing, Polymerase Chain Reaction Testing; 2016. Available from: http://emedicine.medscape.com/article/330178-workup. Accessed August 19, 2016.

21. Centers for Disease Control and Prevention [webpage on the Internet]. Two-step Laboratory Testing Process / Lyme Disease. CDC. Available from: http://www.cdc.gov/lyme/diagnosistesting/labtest/twostep/. Accessed August 19, 2016.

22. Cook M, Puri B. Application of Bayesian decision-making to laboratory testing for Lyme disease and comparison with testing for HIV. Int J Gen Med. 2017;10:113-123.

23. Coulter P, Lema C, Flayhart D, et al. Two-year evaluation of Borrelia burgdorferi culture and supplemental tests for definitive diagnosis of Lyme disease. J Clin Microbiol. 2005;43(10):5080-5084.

24. Wojciechowska-Koszko I, Mączyńska I, Szych Z, Giedrys-Kalemba S. Serodiagnosis of borreliosis: indirect immunofluorescence assay, enzyme-linked immunosorbent assay and immunoblotting. Arch Immunol Ther Exp (Warsz). 2011;59(1):69-77.

25. Schutzer SE, Coyle PK, Belman AL, Golightly MG, Drulle J. Sequestration of antibody to Borrelia burgdorferi in immune complexes in seronegative Lyme disease. Lancet. 1990;335(8685):312-315.

26. Borgermans L, Perronne C, Balicer R, Polasek O, Obsomer V. Lyme disease: time for a new approach? BMJ. 2015;351:h6520.

27. Horowitz RI. Approach to diagnosing Lyme disease misses a large proportion of cases. $B M J .2016 ; 352:$ i1 13 .

28. Ang CW, Notermans DW, Hommes M, Simoons-Smit AM, Herremans T. Large differences between test strategies for the detection of anti-Borrelia antibodies are revealed by comparing eight ELISAs and five immunoblots. Eur J Clin Microbiol Infect Dis. 2011;30(8): $1027-1032$.

29. Marangoni A, Sparacino M, Cavrini F, et al. Comparative evaluation of three different ELISA methods for the diagnosis of early culture-confirmed Lyme disease in Italy. J Med Microbiol. 2005;54(pt 4):361-367.

30. Chmielewski T, Fiett J, Gniadkowski M, Tylewska-Wierzbanowska S. Improvement in the laboratory recognition of Lyme borreliosis with the combination of culture and PCR methods. Mol Diagn. 2003;7(3-4):155-162.

31. Aguero-Rosenfeld ME, Wang G, Schwartz I, Wormser GP. Diagnosis of Lyme borreliosis. Clin Microbiol Rev. 2005;18(3):484-509.

32. Soloski MJ, Crowder LA, Lahey LJ, Wagner CA, Robinson WH, Aucott $\mathrm{JN}$. Serum inflammatory mediators as markers of human Lyme disease activity. PLoS One. 2014;9(4):e93243.

33. Centers for Disease Control and Prevention [webpage on the Internet]. Signs and Symptoms / Lyme Disease. CDC. Available from: http:// www.cdc.gov/lyme/signs_symptoms/. Accessed August 19, 2016.

34. Borgermans L, Goderis G, Vandevoorde J, Devroey D. Relevance of chronic Lyme disease to family medicine as a complex multidimensional chronic disease construct: a systematic review. Int J Family Med. 2014;2014:1-10.

35. Embers ME, Barthold SW, Borda JT, et al. Persistence of Borrelia burgdorferi in rhesus macaques following antibiotic treatment of disseminated infection. PLoS One. 2012;7(1):e29914.

36. Lymedisease.org. Lymedisease.org patient survey results. Available from: https://www.lymedisease.org/wp-content/uploads/2015/04/ lymedisease.org-patient-survey-20151.pdf. Accessed August 19, 2016. 
37. Feder HM, Johnson BJB, O'Connell S, Shapiro ED, Steere AC, Wormser GP. A critical appraisal of "Chronic Lyme Disease.”. N Engl J Med. 2007;357(14):1422-1430.

38. Wormser GP, Dattwyler RJ, Shapiro ED, et al. The clinical assessment, treatment, and prevention of Lyme disease, human granulocytic anaplasmosis, and babesiosis: clinical practice guidelines by the Infectious Diseases Society of America. Clin Infect Dis. 2006;43(9): 1089-1134.

39. Cameron DJ, Johnson LB, Maloney EL. Evidence assessments and guideline recommendations in Lyme disease: the clinical management of known tick bites, erythema migrans rashes and persistent disease. Expert Rev Anti Infect Ther. 2014;12(9):1103-1135.

40. Alexander JM, Cox SM. Lyme disease and pregnancy. Infect Dis Obstet Gynecol. 1995;3(6):256-261.

41. American Red Cross [webpage on the Internet]. American Red Cross Participating in an Investigational Study to Test the Blood Supply for a Tick-Borne Parasite in Donated Blood. Available from: http://www. redcross.org/news/press-release/American-Red-Cross-Participatingin-an-Investigational-Study-to-Test-the-Blood-Supply-for-a-TickBorne-Parasite-in-Donated-Blood. Accessed August 19, 2016.

42. Alexander W. 2012 integrative healthcare symposium: treating the pain of Lyme disease and adopting lifestyle change as therapy. $P T$ Peer Rev J Formul Manag. 2012;37(4):247-249.

43. Horowitz R. Why Can't I Get Better: Solving the Mystery of Lyme \& Chronic Disease. New York, NY: St Martin's Press; 2013.

44. Burrascano J. Advanced Topics in Lyme Disease: Diagnostic Hints and Treatment Guidelines for Lyme and Other Tick Borne Illnesses, 2008. Available from: http://www.lymenet.org/BurrGuide200810.pdf. Accessed August 20, 2016.

45. Wolfe F, Clauw DJ, Fitzcharles M-A, et al. The American College of Rheumatology preliminary diagnostic criteria for fibromyalgia and measurement of symptom severity. Arthritis Care Res. 2010;62(5):600-610.

46. Hahn E, Gottschling J, Spinath FM. Short measurements of personality - validity and reliability of the GSOEP Big Five Inventory (BFI-S). J Res Personal. 2012;46(3):355-359.

47. Klempner MS, Hu LT, Evans J, et al. Two controlled trials of antibiotic treatment in patients with persistent symptoms and a history of Lyme disease. N Engl J Med. 2001;345(2):85-92.

48. Centers for Disease Control and Prevention [webpage on the Internet]. HRQOL-14 - Healthy Days Measure. HRQOL | CDC. Available from: http://www.cdc.gov/hrqol/hrqol14_measure.htm. Accessed August 20, 2016.

49. Shadick NA, Phillips CB, Logigian EL, et al. The long-term clinical outcomes of Lyme disease. A population-based retrospective cohort study. Ann Intern Med. 1994;121(8):560-567.

50. Cronbach LJ. Coefficient alpha and the internal structure of tests. Psychometrika. 1951;16(3):297-334.

51. Nunnally JC. Psychometric Theory. 2nd ed. New York, NY: McGrawHill; 1978.

52. Cortina JM. What is coefficient alpha? An examination of theory and applications. J Appl Psychol. 1993;78(1):98-104.

53. Centers for Disease Control and Prevention [webpage on the Internet]. Case Definitions Overview. NNDSS. Available from: https://wwwn. cdc.gov/nndss/case-definitions.html. Accessed August 20, 2016.

54. Lindberg K. The Oregon Outdoor Recreation Survey. Available from: https://www.oregon.gov/oprd/PLANS/docs/scorp/diversitysurveyinstrument.pdf. Accessed August 20, 2016.

55. Barlow DH, Ellard KK, Sauer-Zavala S, Bullis JR, Carl JR. The origins of neuroticism. Perspect Psychol Sci. 2014;9(5):481-496.

56. Jeronimus BF, Ormel J, Aleman A, Penninx BWJH, Riese H. Negative and positive life events are associated with small but lasting change in neuroticism. Psychol Med. 2013;43(11):2403-2415.

57. Richardson JTE. Eta squared and partial eta squared as measures of effect size in educational research. Educ Res Rev. 2011;6(2):135-147.

58. Cohen J. A power primer. Psychol Bull. 1992;112(1):155-159.
59. de Souza RJ, Eisen RB, Perera S, et al. Best (but oft-forgotten) practices: sensitivity analyses in randomized controlled trials. Am J Clin Nutr. 2016;103(1):5-17.

60. Glass GV, Peckham PD, Sanders JR. Consequences of failure to meet assumptions underlying the fixed effects analyses of variance and covariance. Rev Educ Res. 1972;42(3):237-288.

61. Harwell MR, Rubinstein EN, Hayes WS, Olds CC. Summarizing Monte Carlo results in methodological research: the one- and two-factor fixed effects ANOVA cases. J Educ Behav Stat. 1992;17(4):315-339.

62. Schmider E, Ziegler M, Danay E, Beyer L, Bühner M. Is it really robust? Reinvestigating the robustness of ANOVA against violations of the normal distribution assumption. Methodology. 2010;6(4): 147-151.

63. Kruskal WH, Wallis WA. Use of ranks in one-criterion variance analysis. J Am Stat Assoc. 1952;47(260):583-621.

64. Green SB, Salkind NJ. Using SPSS for Windows and Macintosh: Analyzing and Understanding Data. 7th ed. Boston: Pearson; 2014.

65. Longo DL, editor. Harrison's Principles of Internal Medicine. 18th ed. New York, NY: McGraw-Hill; 2012.

66. Moutailler S, Valiente Moro C, Vaumourin E, et al. Co-infection of ticks: the rule rather than the exception. PLoS Negl Trop Dis. 2016;10(3):e0004539.

67. Branda JA, Linskey K, Kim YA, Steere AC, Ferraro MJ. Two-tiered antibody testing for Lyme disease with use of 2 enzyme immunoassays, a whole-cell sonicate enzyme immunoassay followed by a VlsE C6 peptide enzyme immunoassay. Clin Infect Dis. 2011;53(6):541-547.

68. Ma B, Christen B, Leung D, Vigo-Pelfrey C. Serodiagnosis of Lyme borreliosis by Western immunoblot: reactivity of various significant antibodies against Borrelia burgdorferi. J Clin Microbiol. 1992;30(2):370-376.

69. Rudenko N, Golovchenko M, Grubhoffer L, Oliver JH. Updates on Borrelia burgdorferi sensu lato complex with respect to public health. Ticks Tick Borne Dis. 2011;2(3):123-128.

70. Roscoe C, Epperly T. Tick-borne relapsing fever. Am Fam Physician. 2005;72(10):2039-2044.

71. Anderson A, Bijlmer H, Fournier P-E, et al. Diagnosis and Management of $Q$ Fever - United States, 2013. Available from: https://www. cdc.gov/mmwr/preview/mmwrhtml/rr6203a1.htm. Accessed August $20,2016$.

72. Chowdri HR, Gugliotta JL, Berardi VP, et al. "Borrelia miyamotoi" infection presenting as human granulocytic anaplasmosis: a case report. Ann Intern Med. 2013;159(1):21.

73. Krause PJ, Telford SR, Spielman A, et al. Concurrent Lyme disease and babesiosis. Evidence for increased severity and duration of illness. JAMA. 1996;275(21):1657-1660.

74. Hersh MH, Ostfeld RS, McHenry DJ, et al. Co-infection of blacklegged ticks with Babesia microti and Borrelia burgdorferi is higher than expected and acquired from small mammal hosts. PLoS One. 2014;9(6):e99348.

75. Eleftherios M. When to suspect and how to monitor babesiosis. Am Fam Physician. 2001;63(10):1969-1975.

76. Horowitz ML, Coletta F, Fein AM. Delayed onset adult respiratory distress syndrome in babesiosis. Chest. 1994;106(4):1299-1301.

77. Knapp KL, Rice NA. Human coinfection with Borrelia burgdorferi and Babesia microt in the United States. J Parasitol Res. 2015;2015:1-11.

78. Lempereur L, Shiels B, Heyman P, et al. A retrospective serological survey on human babesiosis in Belgium. Clin Microbiol Infect. 2015;21(1):96.e1-e7.

79. Eskow E, Rao RV, Mordechai E. Concurrent infection of the central nervous system by Borrelia burgdorferi and Bartonella henselae: evidence for a novel tick-borne disease complex. Arch Neurol. 2001;58(9):1357-1363.

80. Pérez-Castrillón JL, Bachiller-Luque P, Martín-Luquero M, MenaMartín FJ, Herreros V. Tularemia epidemic in northwestern Spain: clinical description and therapeutic response. Clin Infect Dis. 2001;33(4):573-576. 
81. Nicolson GL, Gan R, Haier J. Multiple co-infections (Mycoplasma, Chlamydia, human herpes virus-6) in blood of chronic fatigue syndrome patients: association with signs and symptoms. APMIS. 2003;111(5):557-566.

82. Hodzic E, Imai D, Feng S, Barthold SW. Resurgence of persisting non-cultivable Borrelia burgdorferi following antibiotic treatment in mice. PLoS One. 2014;9(1):e86907.

83. Marques A, Telford SR, Turk S-P, et al. Xenodiagnosis to detect Borrelia burgdorferi infection: a first-in-human study. Clin Infect Dis. 2014;58(7):937-945.

84. Zhang Y. Persisters, persistent infections and the Yin-Yang model. Emerg Microbes Infect. 2014;3(1):e3.

85. Feng J, Auwaerter PG, Zhang Y. Drug combinations against Borrelia burgdorferi persisters in vitro: eradication achieved by using daptomycin, cefoperazone and doxycycline. PLoS One. 2015;10(3):e0117207.

86. Horowitz RI. How Can I Get Better? An Action Plan for Treating Resistant Lyme and Chronic Disease. New York, NY: St. Martin's Griffin; 2017.

87. Aucott JN, Soloski MJ, Rebman AW, et al. CCL19 as a chemokine risk factor for posttreatment Lyme disease syndrome: a prospective clinical cohort study. Clin Vaccine Immunol. 2016;23(9):757-766.

88. Steere AC. Lyme disease. N Engl J Med. 2001;345(2):115-125.

89. Sapi E, Balasubramanian K, Poruri A, et al. Evidence of in vivo existence of Borrelia biofilm in borrelial lymphocytomas. Eur J Microbiol Immunol. 2016;6(1):9-24.

90. Merilainen L, Schwarzbach A, Herranen A, Gilbert L. Morphological and biochemical features of Borrelia burgdorferi pleomorphic forms. Microbiology. 2015;161(3):516-527.

91. Timmaraju VA, Theophilus PAS, Balasubramanian K, Shakih S, Luecke DF, Sapi E. Biofilm formation by Borrelia burgdorferi sensu lato. FEMS Microbiol Lett. 2015;362(15):fnv120.

92. Sharma B, Brown AV, Matluck NE, Hu LT, Lewis K. Borrelia burgdorferi, the causative agent of Lyme disease, forms drug-tolerant persister cells. Antimicrob Agents Chemother. 2015;59(8):4616-4624.

93. Horowitz RI, Freeman P. The use of Dapsone as a novel "Persister" drug in the treatment of chronic Lyme disease/post treatment Lyme disease syndrome. J Clin Exp Dermatol Res. 2016;7:3.

94. Centers for Disease Control and Prevention [webpage on the Internet]. Lyme Disease. CDC. Available from: https://www.cdc.gov/lyme/. Accessed August 20, 2016.

95. Christian LM, Glaser R, Porter K, Malarkey WB, Beversdorf D, Kiecolt-Glaser JK. Poorer self-rated health is associated with elevated inflammatory markers among older adults. Psychoneuroendocrinology. 2011;36(10):1495-1504.

96. Kaser A, Zeissig S, Blumberg RS. Inflammatory bowel disease. Annu Rev Immunol. 2010;28(1):573-621.

97. Moscandrew ME, Loftus EV. Diagnostic advances in inflammatory bowel disease (imaging and laboratory). Curr Gastroenterol Rep. 2009;11(6):488-495.

98. Voulgari P. Rheumatological manifestations in inflammatory bowel disease. Ann Gastroenterol. 2011;24(3):173-180.

99. Gaydos CA. Nucleic acid amplification tests for gonorrhea and Chlamydia: practice and applications. Infect Dis Clin North Am. 2005;19(2):367-386, ix.

100. Lohani S, Nazir S, Tachamo N, Patel N. Disseminated gonococcal infection: an unusual presentation. J Community Hosp Intern Med Perspect. 2016;6(3):31841.

101. O’Brien JP, Goldenberg DL, Rice PA. Disseminated gonococcal infection: a prospective analysis of 49 patients and a review of pathophysiology and immune mechanisms. Medicine (Baltimore). 1983;62(6):395-406.

102. Ganem D, Prince AM. Hepatitis B virus infection - natural history and clinical consequences. N Engl J Med. 2004;350(11):1118-1129.
103. Lauer GM, Walker BD. Hepatitis C virus infection. $N$ Engl J Med. 2001;345(1):41-52.

104. McMahon BJ. Natural history of chronic hepatitis B - clinical implications. Medscape J Med. 2008;10(4):91.

105. Nainan OV, Xia G, Vaughan G, Margolis HS. Diagnosis of hepatitis A virus infection: a molecular approach. Clin Microbiol Rev. 2006;19(1): 63-79.

106. Pawlotsky J-M. Molecular diagnosis of viral hepatitis. Gastroenterology. 2002;122(6):1554-1568.

107. Alaedini A, Latov N. Antibodies against OspA epitopes of Borrelia burgdorferi cross-react with neural tissue. J Neuroimmunol. 2005;159(1-2):192-195.

108. Branda JA, Strle F, Strle K, Sikand N, Ferraro MJ, Steere AC. Performance of United States serologic assays in the diagnosis of Lyme borreliosis acquired in Europe. Clin Infect Dis. 2013;57(3): 333-340.

109. Burakgazi AZ. Lyme disease-induced polyradiculopathy mimicking amyotrophic lateral sclerosis. Int J Neurosci. 2014;124(11):859-862.

110. Cimmino MA, Trevisan G. Lyme arthritis presenting as adult onset Still's disease. Clin Exp Rheumatol. 1989;7(3):305-308.

111. Horowitz RI. Clinical roundup: selected treatment options for Lyme disease. Altern Complement Ther. 2012;18(4):220-225.

112. Kologrivova EN, Baraulina AS, Nechaeva SV, et al. [Intensity of the production of rheumatoid factor in patients with different degree of sensitization to Borrelia garinii antigens]. Zh Mikrobiol Epidemiol Immunobiol. 2005;(1):70-72. Russian.

113. Middelveen MJ, Bandoski C, Burke J, et al. Exploring the association between Morgellons disease and Lyme disease: identification of Borrelia burgdorferi in Morgellons disease patients. BMC Dermatol. 2015;15:1.

114. Crowder CD, Carolan HE, Rounds MA, et al. Prevalence of Borrelia miyamotoi in Ixodes ticks in Europe and the United States. Emerg Infect Dis. 2014;20(10):1678-1682.

115. Szer IS, Taylor E, Steere AC. The long-term course of Lyme arthritis in children. N Engl J Med. 1991;325(3):159-163.

116. Thompson A, Mannix R, Bachur R. Acute pediatric monoarticular arthritis: distinguishing Lyme arthritis from other etiologies. Pediatrics. 2009;123(3):959-965.

117. Carter JD, Espinoza LR, Inman RD, et al. Combination antibiotics as a treatment for chronic Chlamydia-induced reactive arthritis: a double-blind, placebo-controlled, prospective trial. Arthritis Rheum. 2010;62(5):1298-1307.

118. Carapetis JR, McDonald M, Wilson NJ. Acute rheumatic fever. Lancet. 2005;366(9480):155-168.

119. Seckeler MD, Hoke T. The worldwide epidemiology of acute rheumatic fever and rheumatic heart disease. Clin Epidemiol. 2011;3:67-84.

120. Guidelines for the diagnosis of rheumatic fever. Jones Criteria, 1992 update. Special Writing Group of the Committee on Rheumatic Fever, Endocarditis, and Kawasaki Disease of the Council on Cardiovascular Disease in the Young of the American Heart Association. JAMA. 1992;268(15):2069-2073.

121. Cojocaru M, Cojocaru IM, Silosi I, Vrabie CD. Manifestations of systemic lupus erythematosus. Maedica (Buchar). 2011;6(4):330-336.

122. Hochberg MC. Updating the American College of Rheumatology revised criteria for the classification of systemic lupus erythematosus. Arthritis Rheum. 1997;40(9):1725.

123. Tan EM, Cohen AS, Fries JF, et al. The 1982 revised criteria for the classification of systemic lupus erythematosus. Arthritis Rheum. 1982;25(11):1271-1277.

124. Weiss NL, Sadock VA, Sigal LH, Phillips M, Merryman PF, Abramson SB. False positive seroreactivity to Borrelia burgdorferi in systemic lupus erythematosus: the value of immunoblot analysis. Lupus. 1995;4(2):131-137. 
The International Journal of General Medicine is an international, peer-reviewed open-access journal that focuses on general and internal medicine, pathogenesis, epidemiology, diagnosis, monitoring and treatment protocols. The journal is characterized by the rapid reporting of reviews, original research and clinical studies across all disease areas.
The manuscript management system is completely online and includes a very quick and fair peer-review system, which is all easy to use. Visit http://www.dovepress.com/testimonials.php to read real quotes from published authors.

Submit your manuscript here: https://www.dovepress.com/international-journal-of-general-medicine-journal 